\title{
Quasi-symmetry effects in the threshold photoelectron spectrum of methyl isocyanate
}

Oliver J. Harper, Laurent H. Coudert, ${ }^{*}$ Bérenger Gans, Stéphane Douin, and Séverine Boyé-Péronne Université Paris-Saclay, CNRS, Institut des Sciences Moléculaires d'Orsay, 91405, Orsay, France

Jean-Christophe Loison

Institut des Sciences Moléculaires, UMR 5255 CNRS - Université de Bordeaux, Bât. A12, 351 Cours de la Libération, F-33405 Talence cedex, France

Gustavo A. Garcia

L'Orme des Merisiers, Saint Aubin BP 48, Synchrotron SOLEIL, Gif sur Yvette, France

Jean-Claude Guillemin

Univ. Rennes, Ecole Nationale Supérieure de Chimie de Rennes, CNRS, ISCR-UMR6226, F-35000 Rennes, France

(Dated: September 2, 2020)

\begin{abstract}
The vacuum-ultraviolet threshold photoelectron spectrum of methyl isocyanate $\mathrm{CH}_{3} \mathrm{NCO}$ has been recorded from 10.4 to $12 \mathrm{eV}$ using synchrotron radiation and a coincidence technique allowing for a mass-discrimination of the photoelectron signal. A significant improvement is achieved over previous investigations as this experimental setup leads to a much more resolved spectrum. Ten sharp peaks and a broad feature spanning $1.2 \mathrm{eV}$ were recorded. This spectrum consists of the $\tilde{X}^{+2} A^{\prime \prime} \leftarrow \tilde{X}^{1} A^{\prime}$ and $\tilde{A}^{+2} A^{\prime} \leftarrow \tilde{X}^{1} A^{\prime}$ ionizing transitions. For the former, the adiabatic ionization energy was determined experimentally to be $10.596(6) \mathrm{eV}$; for the latter its value was estimated to be $10.759(50) \mathrm{eV}$. Seven sharp peaks could be assigned to vibrational modes of the cation $\tilde{X}^{+2} A^{\prime \prime}$ and neutral $\tilde{X}^{1} A^{\prime}$ ground electronic states involving only the NCO group atoms. Theoretical modeling of the threshold photoelectron spectrum has proven difficult as methyl isocyanate is a non-rigid molecule displaying large amplitude internal rotation of the methyl group and $\angle \mathrm{CNC}$ bending mode, leading to the quasi-symmetry. With the help of $a b$ initio calculations, a theoretical model in which these two large amplitude motions are included in addition to the five small amplitude vibrational modes involving NCO group atoms is proposed. Comparison with the experimental spectrum shows that the broad feature and the strongest peaks line positions are well accounted for; their intensities are also fairly well reproduced after adjusting a few parameters.
\end{abstract}

\section{INTRODUCTION}

Methyl isocyanate $\left(\mathrm{CH}_{3} \mathrm{NCO}\right)$ is a reactive chemical compound $^{1}$ which is an intermediate in the industrial production of carbamate. It is widely used in the synthesis of rubbers, adhesives and pesticides, and was the main toxicant associated with the Bhopal disaster. Its ground electronic state is spectroscopically well characterized as it is a benchmark molecule for quasi-symmetric species. Such species, of the $\mathrm{WH}_{3} \mathrm{XYZ}$ type, display two large amplitude motions, the $\mathrm{WH}_{3}$ torsion and $\angle \mathrm{WXY}$ bending mode, leading to a singularity at the symmetrical $C_{3 v}$ configuration. Dedicated theoretical treatments developed to evaluate the rotation-bending-torsion energy levels of such species ${ }^{2-11}$ were applied to the analysis of the microwave spectrum of methyl isothiocyanate ${ }^{3,12}$ $\left(\mathrm{CH}_{3} \mathrm{NCS}\right)$, silyl isocyanate ${ }^{5,7}\left(\mathrm{SiH}_{3} \mathrm{NCO}\right)$, and methyl isocyanate. ${ }^{8,12-14}$ For the latter species, nearly 400 transitions could be assigned leading to its detection in the interstellar space medium. ${ }^{14,15}$ It was then believed to be one of the most abundant molecules after water and

* laurent.coudert@u-psud.fr formamide on the surface of the comet $67 \mathrm{P} /$ ChuryumovGerasimenko, ${ }^{16}$ but this was later disputed. ${ }^{17}$

In order to further investigate the effects of quasisymmetry, spectroscopic investigations of the infrared spectrum of methyl isocyanate ${ }^{18,19}$ were carried out and the two low energy vibrations corresponding to the large amplitude methyl group torsion and $\angle \mathrm{CNC}$ bending mode were observed. The photoabsorption cross section and fluorescence excitation spectra were measured by Tokue et $a l .{ }^{20}$ in the $105-210 \mathrm{~nm}$ range, and three Rydberg series were assigned with their vibrational progressions. This analysis provided a value of the ionization energy of $10.674 \mathrm{eV}$. Above the ionization energy $(I E)$, fewer laboratory experiments have been performed and little information is currently available on the cation $\mathrm{CH}_{3} \mathrm{NCO}^{+}$. Photoelectron spectra were recorded ${ }^{21,22}$ with a HeI lamp with a typical resolution of $15 \mathrm{meV}$. From 10 to $18 \mathrm{eV}$, seven bands were observed and unambiguously assigned in terms of NCO group orbitals. The two lowest lying bands, in the 10.4 to $12 \mathrm{eV}$ energy range, display distinct spectroscopic features and the first vertical ionization energy was measured ${ }^{22}$ at $10.63 \mathrm{eV}$. No attempts were made to understand the photoelectron spectrum in this energy range accounting for the quasisymmetry effects. 
In the present work, the experimental threshold photoelectron spectrum (TPES) of methyl isocyanate has been recorded from 10.4 to $12 \mathrm{eV}$ using synchrotron radiation. This spectrum, characterized by a better resolution than those previously reported, ${ }^{21,22}$ displays several sharp lines and a broad feature. It has been modeled with two theoretical treatments accounting for quasi-symmetry effects. In the first treatment, only the two large amplitude motions are considered..$^{2,3,5,7-11}$ In the second one, making use of the harmonic adiabatic approximation, ${ }^{23,24}$ the previous treatment is extended to include small amplitude vibrational modes. In agreement with Eland, ${ }^{25}$ only the five small amplitude vibrational modes involving the NCO group are included because this group undergoes the largest structural changes upon ionization.

The paper has four remaining sections. In Section II, the experimental setup and results are described. Section III reports on the calculation of the bending-torsion and vibration-bending-torsion energy levels. Section IV deals with the simulated TPES and comparisons with the experimental one. Section $\mathrm{V}$ is the conclusion.

\section{EXPERIMENTAL}

\section{A. $\mathrm{CH}_{3} \mathrm{NCO}$ TPES and photoion yield recording}

Methyl isocyanate was prepared with a modified version of the synthesis of Han et al. ${ }^{26}$ as reported in Maté et $a .^{27} \mathrm{~A}$ few grams of the sample were placed in a vial attached to the experiment. The vial was plunged into a chiller set at $-40^{\circ} \mathrm{C}$ to lower the vapor pressure to around $10^{-1}$ mbar, which was otherwise $280 \mathrm{mbar}$ at $0^{\circ} \mathrm{C}$. This step was necessary to avoid too great a build-up of the sample in the experiment which could lead to polymerization or blockages, but also to limit the consumption of the sample. Maintaining the sample at a constant temperature also provided pressure stability. The gaseous molecules were fed into the SAPHIRS endstation $^{28}$ through a nozzle $30 \mu \mathrm{m}$ in diameter. The beam was then doubly skimmed and irradiated at a right angle by the tunable synchrotron VUV radiation from the DESIRS beamline ${ }^{29}$ at the French national synchrotron facility SOLEIL. The spectral purity of the photons was ensured by a gas filter located upstream from the beamline monochromator and filled with argon. ${ }^{30}$ Argon absorption lines appearing in the ion yield were used to calibrate the photon energy scale. All experimental spectra are corrected by the photon flux, which was monitored as a function of the photon energy with an Si photodiode (AXUV, IRD).

After the photoionization of the neutral species by the monochromatized synchrotron radiation, the resulting photoelectrons and photoions were analyzed with the DELICIOUS III spectrometer. ${ }^{31}$ The photoion yield (PIY) of $\mathrm{CH}_{3} \mathrm{NCO}^{+}(\mathrm{m} / q=57$ a.m.u $)$ was recorded with a photon resolution of $5 \mathrm{meV}$ using a $177 \mathrm{~V} / \mathrm{cm}$ extrac- tion field to ensure that all ions associated with electrons possessing a kinetic energy up to $7 \mathrm{eV}$ were collected.

The TPES spectrum was obtained using the slow photoelectron spectroscopy (SPES) technique, ${ }^{32}$ in which electrons whose kinetic energy is less than $5 \mathrm{meV}$ are taken into account. The total resolution (electron + photon) was $8 \mathrm{meV}\left(65 \mathrm{~cm}^{-1}\right)$ and the absolute accuracy of the energy scale was found to be $3.2 \mathrm{meV}$. Note that the $53 \mathrm{~V} / \mathrm{cm}$ DC field used to extract the photoelectrons resulted in a field-induced shift ${ }^{33}$ of the ionization energy of about $-5.4 \mathrm{meV}$. Hence all ionization energies given in the following sections are corrected from the field-induced shift. The final absolute accuracy on ionization threshold measurements was about $6 \mathrm{meV}$.

\section{B. Experimental results}

Figure 1 displays the TPES of $\mathrm{CH}_{3} \mathrm{NCO}$ and the PIY of $\mathrm{CH}_{3} \mathrm{NCO}^{+}$, recorded from 10.4 to $12 \mathrm{eV}$. The PIY displays a sharp rise around $10.6 \mathrm{eV}$ followed by a smooth increase. It then plateaus and even slightly decreases from roughly $11.5 \mathrm{eV}$. This energy coincides with the appearance of other fragments in the mass spectrum. The strongest signal appearing around $12 \mathrm{eV}$ is the $\mathrm{m} / q=$ 56 a.m.u. fragment, which is assigned to $\mathrm{CH}_{2} \mathrm{NCO}^{+}$produced via dissociative ionization of the parent molecule through loss of $\mathrm{H}$. The $m / q=57$ a.m.u. ion yield does not present any observable autoionization features, hence the intensities of the TPES peaks are probably not affected by resonant autoionization signals.

The TPES consists of the $\tilde{X}^{+2} A^{\prime \prime} \leftarrow \tilde{X}^{1} A^{\prime}$ and $\tilde{A}^{+2} A^{\prime} \leftarrow \tilde{X}^{1} A^{\prime}$ ionizing transitions, ${ }^{21,22}$ where $\tilde{X}^{1} A^{\prime}$, $\tilde{X}^{+2} A^{\prime \prime}$, and $\tilde{A}^{+2} A^{\prime}$ are the neutral ground electronic state and the cation ground and first excited electronic states, respectively. As shown in Fig. 1, the experimental TPES displays a broad feature, from 10.6 to $11.8 \mathrm{eV}$, and 10 sharp peaks. Their Stark-shift corrected ionization energies are given in Table I; peak \# 2 at $10.596 \mathrm{eV}$ is the strongest. For this peak, the ionization energy measured by Pasinszki et al. ${ }^{22}$ with conventional photoelectron spectroscopy is $10.630(15) \mathrm{eV}$ which is $34 \mathrm{meV}$ higher. Assuming that the remaining peaks are the first members of a vibrational Franck-Condon progression, they were tentatively assigned to vibrational modes of the neutral species and cation $\tilde{X}^{+2} A^{\prime \prime}$ ground electronic state. These assignments, given in Table I, are corroborated by the calculated vibrational frequencies of Section III C. We can see that observed and calculated fundamental vibrational frequencies are at most within $93 \mathrm{~cm}^{-1}$. For $\nu_{1}^{\prime}, \nu_{2}^{\prime}$, and $\nu_{3}^{\prime}$, Pasinszki et al. ${ }^{22}$ reported experimental values of 2150,1290 , and $800 \mathrm{~cm}^{-1}$ which differ from the present experimental values. 


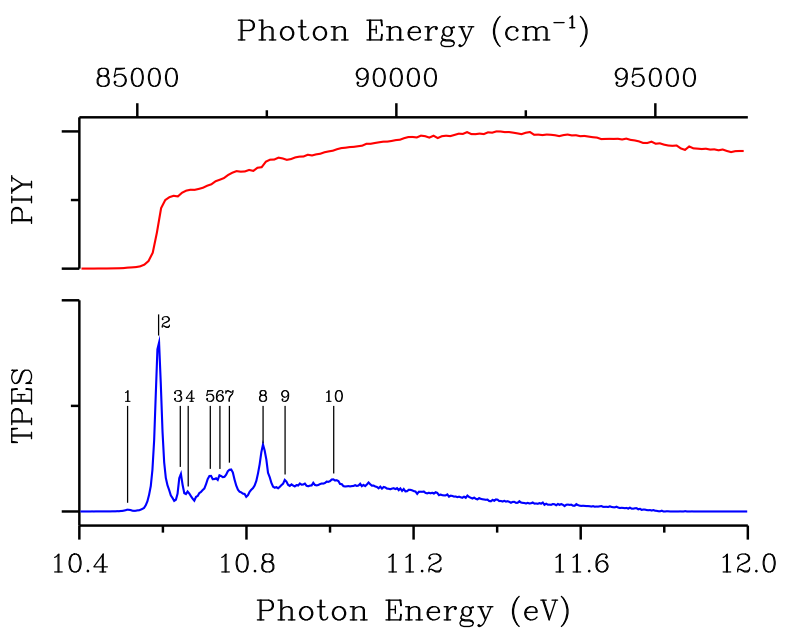

Figure 1. The experimental TPES of the $\tilde{X}^{+2} A^{\prime \prime} \leftarrow \tilde{X}^{1} A^{\prime}$ and $\tilde{A}^{+2} A^{\prime} \leftarrow \tilde{X}^{1} A^{\prime}$ ionizing transitions of methyl isocyanate and the PIY signal are plotted as function of the photon energy in the lower and upper panels, respectively. For the TPES, vertical lines indicate the position of the 10 strongest sharp peaks numbered from 1 to 10 .

\section{THE MODELS}

In the present investigation, the harmonic adiabatic approximation is used. ${ }^{23,24}$ The internal coordinates $q_{i}$ consist of $n$ active large amplitude coordinates with $1 \leq i \leq n$ and of $m$ inactive small amplitude coordinates with $n+1 \leq i \leq n+m$. These coordinates are chosen in this section and, with the help of ab initio calculations, Eqs. (4a) and (4b) of Lauvergnat et al. ${ }^{23}$ are solved allowing us to retrieve $q_{i}^{\text {eq }}\left(q_{j}\right)$ the equilibrium values of the inactive coordinates as functions of the active coordinates. The Hessian matrix, also retrieved from $a b$ initio calculations, is parameterized with the active coordinates. This second step leads to the normal modes and fundamental frequencies describing the harmonic oscillator energy levels associated with the inactive coordinates. The anharmonic potential is then obtained, also from $a b$ initio results. These numerical results allow us to retrieve energy levels for the neutral and the cation using two different theoretical approaches.

\section{A. Ab initio calculation results}

There are 15 internal coordinates in methyl isocyanate which are defined in Table II. Coordinates $q_{i}$, with $3 \leq$ $i \leq 6$ and $8 \leq i \leq 13$, are expressed in terms of bond lengths, bond angles, and dihedral angles using the atom labeling of Fig. 2. Coordinate $q_{7}=\theta$ is the out-of-plane bending angle of the NO direction with respect to the $\mathrm{C}_{\alpha} \mathrm{NC}$ plane. ${ }^{34,35}$ Coordinates $q_{1}=\tau, q_{2}=\rho, q_{14}=\beta_{1}$,
Table I. Peak energy ${ }^{\mathrm{a}}$ and assignment

\begin{tabular}{crrcc}
\hline \hline Peak \# & $E / \mathrm{eV}$ & $\Delta E / \mathrm{cm}^{-1}$ & Assignment $^{\mathrm{b}}$ & $\tilde{\nu}(\mathrm{Cal}) / \mathrm{cm}^{-1}$ \\
\hline 1 & 10.521 & -604 & $\nu_{4}^{\prime \prime}$ & $621^{\mathrm{c}}$ \\
2 & 10.596 & 0 & & \\
3 & 10.648 & 420 & & \\
4 & 10.665 & 555 & $\nu_{4}^{\prime}$ & 541 \\
5 & 10.719 & 986 & $\nu_{3}^{\prime}$ & $920^{\mathrm{d}}$ \\
6 & 10.743 & 1186 & & \\
7 & 10.767 & 1374 & $\nu_{2}^{\prime}$ & $1338^{\mathrm{d}}$ \\
8 & 10.846 & 2011 & $\nu_{1}^{\prime}$ & $2104^{\mathrm{d}}$ \\
9 & 10.898 & 2430 & $2 \nu_{2}^{\prime}$ & 2658 \\
10 & 11.015 & 3376 & & \\
\hline \hline
\end{tabular}

a The Stark-shift corrected ionization energy is given in the column headed $E$; the relative energy with respect to the main peak, peak $\# 2$, is given in the column headed $\Delta E$.

b Assignment in terms of the vibrational modes defined in Section III C is given in this column; calculated frequency in the column headed $\tilde{\nu}$. A prime (double prime) denotes a vibrational mode of the cation (neutral species) ground electronic state.

c This peak is a hot band originating from an excited vibrational state of the neutral species.

${ }^{\mathrm{d}}$ For $\nu_{1}^{\prime}, \nu_{2}^{\prime}$, and $\nu_{3}^{\prime}$, the observed values reported in Ref. ${ }^{22}$ are 2150,1290 , and $800 \mathrm{~cm}^{-1}$, respectively.

and $q_{15}=\beta_{2}$ are four angles:

$$
\begin{array}{ll}
\tau=\left(\alpha_{1}+\alpha_{2}+\alpha_{3}-\pi\right) / 3, & \rho=\pi-\angle \mathrm{C}_{\alpha} \mathrm{NC}, \\
\beta_{1}=2 \alpha_{1}-\alpha_{2}-\alpha_{3}, & \beta_{2}=\alpha_{2}-\alpha_{3},
\end{array}
$$

where $\alpha_{i}$ is the dihedral angle $\angle \mathrm{CNC}_{\alpha} \mathrm{H}_{i}$ for hydrogen atom $\mathrm{H}_{i}$. Equations (1) emphasize that $\tau$ and $\rho$ correspond respectively to the internal rotation of the methyl group and to the $\angle \mathrm{C}_{\alpha} \mathrm{NC}$ bending mode. Irrespective of $\tau$, the angles $\beta_{1}$ and $\beta_{2}$ parameterize the distortion of the methyl group from $C_{3 v}$ symmetry. For an undistorted methyl group $\beta_{1}$ is 0 and $\beta_{2}$ is either $120^{\circ}$ or $240^{\circ}$. Coordinates $q_{1}=\tau$ and $q_{2}=\rho$ are chosen as the active coordinates; the remaining coordinates $q_{i}$, with $3 \leq i \leq 15$, are the inactive coordinates.

The $a b$ initio calculations were performed using the CCSD(T) (Coupled-Cluster with Single and Double and perturbative Triple excitations) method explicitly correlated (RCCSD(T)-F12) using the MOLPRO 2012 package $^{36}$ and the Dunning augmented triple zeta basis (AVTZ). Still using the same method, $\operatorname{RCCSD}(\mathrm{T})$ F12/AVTZ, various sets of calculations have been performed to compute the potential energy surfaces (PES) of $\tilde{X}^{1} A^{\prime}, \tilde{X}^{+2} A^{\prime \prime}$, and $\tilde{A}^{+2} A^{\prime}$ states in reduced coordinates so as to determine the parameters needed to analyze the spectra. These PES are the bending potential energy curves as a function of the $\mathrm{C}_{\alpha} \mathrm{NC}$ bending angle, where all other parameters were optimized, the vibrational frequencies being calculated at each angle value. For several values of this bending angle, the Hessian has also been calculated to obtain Wilson's $\mathbf{F}$ matrix. ${ }^{34}$ Finally, the bending-torsion potential of the $\mathrm{CH}_{3}$ group was 


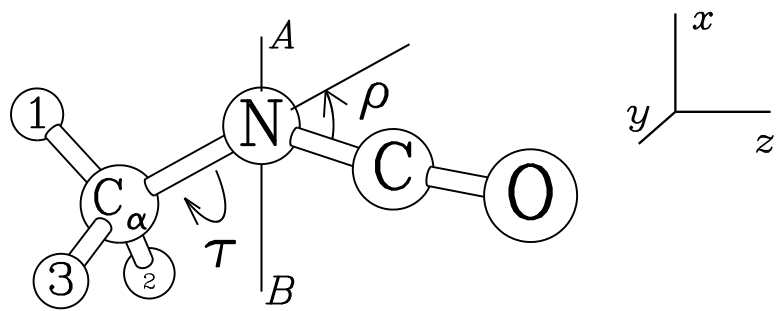

Figure 2. Atom labeling in methyl isocyanate. The numbers 1,2 , and 3 identify the three hydrogen atoms of the methyl group, its carbon atom being labeled $\mathrm{C}_{\alpha} . \tau$ and $\rho$ are the two active large amplitude coordinates corresponding to the internal rotation of the methyl group and the $\angle \mathrm{C}_{\alpha} \mathrm{NC}$ bending mode. The molecule fixed $x y z$ axis system is shown. For clarity its origin is not drawn at the molecular center of mass. The $A B$ axis bisects the $\angle \mathrm{C}_{\alpha} \mathrm{NC}$ angle and is parallel to the $x$ axis.

calculated again by optimizing all other parameters including the $\mathrm{C}_{\alpha}-\mathrm{H}$ bonds.

\section{Equilibrium structures}

The equilibrium structures of the neutral and the cationic species were determined in the first set of $a b i n i$ tio calculations. The 3 equilibrium structures are drawn in Fig. 3. Equilibrium values for the internal coordinates are given in Table II where, in the case of the neutral, they are compared to those retrieved from the analysis of the microwave spectrum. ${ }^{8}$ For the neutral species and the cation electronic ground states, a $C_{s}$ bent structure arises with hydrogen atom $\mathrm{H}_{1}$ in the symmetry plane and pointing away from the NCO group. The value of $\beta_{2}$ indicates that the methyl group is distorted from $C_{3 v}$ symmetry, especially for the cation $\tilde{X}^{+2} A^{\prime \prime}$ state. For the neutral ground state, the agreement with the experimental equilibrium values ${ }^{8}$ is better than $1 \%$ except for the angles $q_{2}=\rho$ and $q_{6}=\angle \mathrm{NCO}$. For the cation $\tilde{A}^{+2} A^{\prime}$ state, a nearly linear structure with almost $C_{3 v}$ symmetry arises. The value of $\beta_{2}$ indicates a more distorted methyl group than in the two previous cases.

\section{Determination of $q_{i}^{\mathrm{eq}}(\tau, \rho)$}

The equilibrium values of the 13 inactive coordinates are approximated with analytical expansions $F_{i}(\tau, \rho)$ compatible with the symmetry of the molecule and depending on the two active coordinates $\tau$ and $\rho$ :

$$
q_{i}^{\mathrm{eq}}(\tau, \rho) \approx F_{i}(\tau, \rho)=q_{i}^{0}+\sum_{j=1}^{n} g_{j}^{i}(\tau) \rho^{j},
$$

where $q_{i}^{0}$ is the value of $q_{i}^{\text {eq }}$ for the $\rho=0, C_{3 v}$ configuration and $g_{j}^{i}(\tau)$ is a Fourier-type expansion. The values of $q_{i}^{0}$ and the expansions $g_{j}^{i}(\tau)$ can be found in

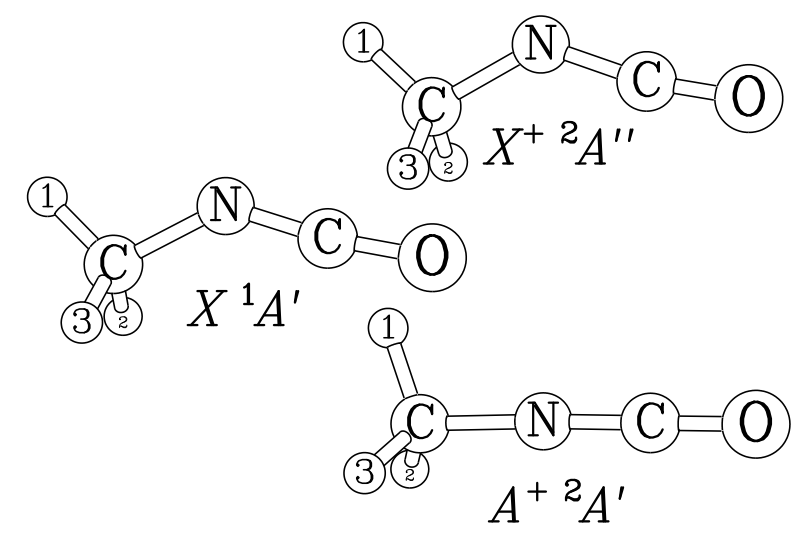

Figure 3. The equilibrium structures obtained in Section III A 1 for the neutral $\tilde{X}^{1} A^{\prime}$ electronic ground state and the cation $\tilde{X}^{+2} A^{\prime \prime}$ and $\tilde{A}^{+2} A^{\prime}$ lowest electronic states.

Eqs. (A1)-(A6). The $F_{i}(\tau, \rho)$ expansions were numerically retrieved from the second set of ab initio calculations in which $\rho$ was constrained to several values ranging from $0.1^{\circ}$ to $80^{\circ}$. Convergence, however, could only be attained for one $\tau$-value. For the neutral species and the cation ground states, results are only available for $\tau=0$; for the cation $\tilde{A}^{+2} A^{\prime}$ excited electronic state, only for $\tau=60^{\circ}$. These two $\tau$ values being (within $\pm 2 \pi / 3$ ) those characterizing the corresponding equilibrium configuration in Table II. This prevents us from retrieving the $\tau$ dependence of the expansions in Eq. (2) and we were led to use simplified expansions $F_{i}(\rho)$ with no $\tau$-dependence. These expansions are expressed in terms of fitted parameters $f_{j}^{i}$. For the three bond lengths $q_{i}$, with $3 \leq i \leq 5$, $F_{i}(\rho)$ is an even function of $\rho$ :

$$
F_{i}(\rho)=q_{i}^{0}+\sum_{j=1}^{n} f_{j}^{i} \rho^{2 j}
$$

For the bond angle $q_{6}=\angle \mathrm{NCO}$ and the angle $q_{15}=\beta_{2}$, there is no parity restriction:

$$
F_{i}(\rho)=q_{i}^{0}+\sum_{j=1}^{n} f_{j}^{i} \rho^{j} .
$$

For the angles $q_{7}=\theta$ and $q_{14}=\beta_{1}$, there is no $\rho$ dependence and $F_{i}(\rho)=q_{i}^{0}$. For the 6 coordinates $q_{i}$, with $8 \leq i \leq 13$, parameterizing the methyl group, Eq. (4) can be used. For the three bond lengths $q_{i}$, with $8 \leq i \leq 10$, the relations $f_{j}^{8}=f_{j}^{9}=f_{j}^{10}$, for $j$ even, and $f_{j}^{8}=-f_{j}^{9}=-f_{j}^{10}$, for $j$ odd, hold. Similar relations are fulfilled by the three bond angles $q_{i}$, with $11 \leq i \leq 13$.

The fit of the $a b$ initio data showed that the inactive coordinates $q_{i}$, with $8 \leq i \leq 15$, describing the methyl group, display little dependence on the angle $\rho$. A larger dependence was observed for the inactive coordinates $q_{i}$, with $3 \leq i \leq 7$, describing the NCO group. Figures 4 and 5 show the results of the fitting for two such coordinates, $q_{3}=r(\mathrm{CN})$ and $q_{6}=\angle \mathrm{NCO}$. It can be seen that the 
Table II. Expressions ${ }^{\mathrm{a}}$ and equilibrium values ${ }^{\mathrm{b}}$ of the internal coordinates for the ground electronic state of the neutral and the two lowest electronic states of the cationic species.

\begin{tabular}{lrrrr}
\hline \hline & \multicolumn{2}{c}{$\tilde{X}^{1} A^{\prime}$} & $\tilde{X}^{+2} A^{\prime \prime}$ & $\tilde{A}^{+2} A^{\prime}$ \\
\cline { 2 - 4 } Coordinates & This work & \multicolumn{1}{c}{ Ref. $^{8}$} & & \\
\hline$q_{1}=\tau$ & 0.0 & 0.0 & 0.0 & 180.1 \\
$q_{2}=\rho$ & 44.3 & 39.8 & 48.1 & 1.9 \\
$q_{3}=r(\mathrm{CN})$ & 1.206 & 1.207 & 1.263 & 1.209 \\
$q_{4}=r(\mathrm{CO})$ & 1.176 & 1.171 & 1.145 & 1.165 \\
$q_{5}=r\left(\mathrm{C}_{\alpha} \mathrm{N}\right)$ & 1.447 & 1.434 & 1.436 & 1.417 \\
$q_{6}=\angle \mathrm{NCO}$ & 172.9 & 180.0 & 170.4 & 179.8 \\
$q_{7}=\theta$ & 0.0 & 0.0 & 0.0 & 0.0 \\
$q_{8}=r\left(\mathrm{C}_{\alpha} \mathrm{H}_{1}\right)$ & 1.088 & 1.091 & 1.085 & 1.107 \\
$q_{9}=r\left(\mathrm{C}_{\alpha} \mathrm{H}_{2}\right)$ & 1.091 & 1.091 & 1.102 & 1.091 \\
$q_{10}=r\left(\mathrm{C}_{\alpha} \mathrm{H}_{3}\right)$ & 1.091 & 1.091 & 1.102 & 1.091 \\
$q_{11}=\angle \mathrm{NC}_{\alpha} \mathrm{H}_{1}$ & 108.5 & 108.4 & 108.6 & 104.8 \\
$q_{12}=\angle \mathrm{NC}_{\alpha} \mathrm{H}_{2}$ & 110.6 & 110.0 & 108.4 & 109.6 \\
$q_{13}=\angle \mathrm{NC}_{\alpha} \mathrm{H}_{3}$ & 110.6 & 110.0 & 108.4 & 109.6 \\
$q_{14}=\beta_{1}$ & 0.0 & 0.0 & 0.0 & 0.0 \\
$q_{15}=\beta_{2}$ & 121.4 & 120.7 & 115.0 & 233.0 \\
\hline \hline
\end{tabular}

a Internal coordinates are defined with the atom labeling of Fig. 2. $\tau, \rho, \beta_{1}$, and $\beta_{2}$ are 4 angles defined in Eqs. (1); $\theta$ is the out-of-plane bending angle of the oxygen atom. ${ }^{34,35}$

b Bond lengths are in $\AA$ and angles in degrees.

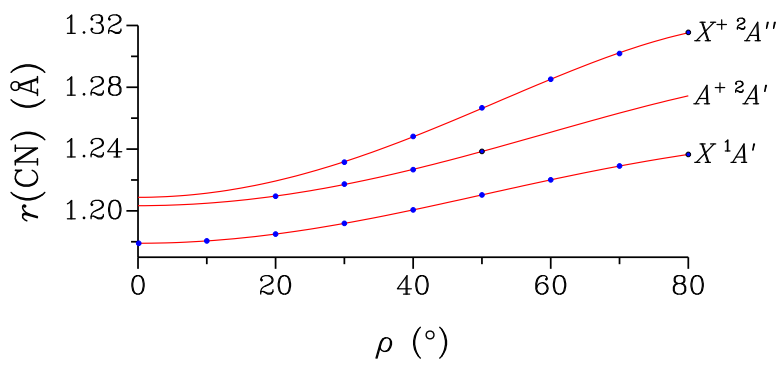

Figure 4. Variations with the active large amplitude coordinate $\rho$ of the bond length $r(\mathrm{CN})$ for the neutral $\tilde{X}^{1} A^{\prime}$ ground electronic state and the cation $\tilde{X}^{+2} A^{\prime \prime}$ and $\tilde{A}^{+2} A^{\prime}$ electronic states. $A b$ initio values are indicated by dots. Solid lines are fitted values calculated with Eq. (3).

$a b$ initio results are well reproduced by the expansions in Eqs. (3) and (4).

\section{Normal modes and vibrational frequencies}

The $F G$ method $^{34}$ was utilized to derive normal modes and fundamental vibrational frequencies from the harmonic expansion of the potential energy. Cartesian coordinates of the Hessian matrix were extracted from the third set of $a b$ initio calculations allowing us to obtain Wilson's $\mathbf{F}$ matrix. Due to the limited set of data, its $\tau$ dependence was ignored and a cubic spline interpolation

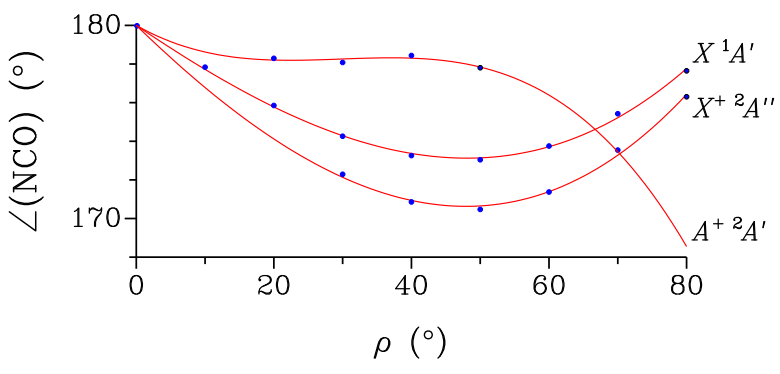

Figure 5. Variations with the active large amplitude coordinate $\rho$ of the bond angle $\angle \mathrm{NCO}$ for the neutral $\tilde{X}^{1} A^{\prime}$ ground electronic state and the cation $\tilde{X}^{+2} A^{\prime \prime}$ and $\tilde{A}^{+2} A^{\prime}$ electronic states. Ab initio values are indicated by dots. Solid lines are fitted values calculated with Eq. (4).

was used to obtain its $\rho$-dependence. Care was taken to have the correct behavior near the $\rho=0, C_{3 v}$ configuration.

As stressed in the introduction, the $F G$ method was applied to a reduced set of inactive coordinates. The 5 selected coordinates $q_{i}$ are those with $3 \leq i \leq 7$, involving NCO group atoms. ${ }^{25} \mathbf{A} \mathbf{B}(\rho)$ matrix $^{\overline{34}}$ was calculated using Section III A 2 leading to a $5 \times 5 \mathbf{G}(\rho)$ matrix. Solving the vibrational problem leads to normal modes, fundamental vibrational frequencies $\nu_{n}(\rho)$, and an $\mathbf{L}(\rho)$ matrix. The latter relates the normal coordinates $Q_{n}$ to the internal coordinates by $q_{i}-q_{i}^{\mathrm{eq}}(\rho)=\sum_{n} L_{i, n}(\rho) Q_{n}$. The wavefunction of the harmonic oscillators, depending parametrically on the active coordinate $\rho$, is written:

$$
\Psi_{\mathbf{n}}(\mathbf{Q} ; \rho)
$$

where the subscripted $\mathbf{n}$ is a shorthand notation for the 5 vibrational quantum numbers $n_{1}, \ldots, n_{5}$. For the cation $\tilde{A}^{+2} A^{\prime}$ state, Fig. 6 shows the variations of the five fundamental frequencies with $\rho$. There are four $A^{\prime}$ vibrational modes $\nu_{n}$, with $1 \leq n \leq 4$, corresponding respectively to $\nu_{a}(\mathrm{NCO})$ the antisymmetrical stretch, $\nu_{s}(\mathrm{NCO})$ the symmetrical stretch, the $\mathrm{C}_{\alpha}-\mathrm{N}$ stretch, and the in-plane $\angle \mathrm{NCO}$ bend. The only $A^{\prime \prime}$ vibrational mode $\nu_{5}$ corresponds to the out-of-plane $\angle \mathrm{NCO}$ bend. Near $\rho=0$, Fig. 6 shows that $\tilde{\nu}_{4}$ and $\tilde{\nu}_{5}$ become close to each other as they correlate to a doubly degenerate vibrational mode in the $C_{3 v}$ configuration.

\section{Anharmonic potential}

The anharmonic potential is the part of the potential depending only on the active coordinates. In agreement with previous investigations of quasi-symmetric top molecules, ${ }^{7-9}$ we take:

$$
V_{0}(\tau, \rho)=V_{b}(\rho)-\frac{1}{2} V_{3}^{0} \rho \cos 3 \tau,
$$

where $V_{b}(\rho)$ is the pure bending potential and the term $\frac{1}{2} V_{3}^{0} \rho \cos 3 \tau$, involving the parameter $V_{3}^{0}$, is a 3 -fold torsional potential with a $\rho$-dependent barrier equal to $\rho V_{3}^{0}$. 


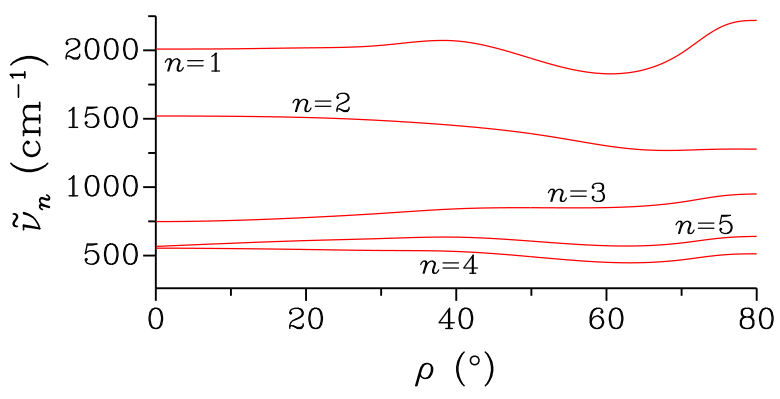

Figure 6. For the cation $\tilde{A}^{+2} A^{\prime}$ electronic state, variations with $\rho$ of the five fundamental frequencies $\tilde{\nu}_{n}(\rho)$, with $1 \leq n \leq$ 5 . Each curve is identified by $n$. See text for a description of the inactive coordinates used.

The pure bending potentials were determined in the last set of $a b$ initio calculations where the zero-point energy was included. The $a b$ initio energies were fitted to the expansion in Eq. (3). Figure 7 shows the variations with $\rho$ of the bending potentials obtained for the neutral and the cationic species. The bending potential retrieved for the neutral from the analysis of the microwave spectrum ${ }^{8}$ is also shown. Figure 7 is consistent with the results in Section III A 1 for the equilibrium $\rho$-values. In agreement with Cradock et al. ${ }^{21}$ and Pasinszki et al. ${ }^{22}$ this figure also shows that the $\tilde{X}^{+2} A^{\prime \prime}$ and $\tilde{A}^{+2} A^{\prime}$ electronic states of the cationic species become degenerate for the $\rho=0, C_{3 v}$ configuration. The barriers to linearity extracted from this last set of $a b$ initio calculations are 841 and $1181 \mathrm{~cm}^{-1}$ for the neutral and the cationic species, respectively. For the neutral species, the barrier to linearity is smaller than that obtained from the analysis of the microwave spectrum, ${ }^{8} 1048 \mathrm{~cm}^{-1}$. The mismatch between the experimental ${ }^{8}$ and the present $a b$ initio potential might be due to model differences. Koput ${ }^{8}$ assumed a rigid linear NCO group while its structure was optimized in the present calculation as indicated by Figs. 4 and 5 .

The bending-torsional parameter $V_{3}^{0}$ in Eq. (6), also determined in this last set of $a b$ initio calculations, is $10.3,125.6$, and $-432.6 \mathrm{~cm}^{-1} / \mathrm{rad}$ for the neutral $\tilde{X}^{1} A^{\prime}$ state and the cation $\tilde{X}^{+2} A^{\prime \prime}$ and $\tilde{A}^{+2} A^{\prime}$ states, respectively. These results are consistent with the equilibrium $\tau$-values in Table II. For the neutral species, the analysis of the microwave spectrum ${ }^{8}$ yielded a value of $20 \mathrm{~cm}^{-1} / \mathrm{rad}$ which is twice as large as that obtained in this work.

\section{B. Bending-torsion energy levels}

The zeroth-order rotation-bending-torsion Hamiltonian $H_{\text {rbt }}$ depends on the Eulerian-type angles, describing the overall rotation, and on the two active large amplitude coordinates. Following Wierzbicki et al. ${ }^{4}$ and Koput, ${ }^{8}$ we choose a reference configuration represented by atom positions $\mathbf{a}_{k}(\tau, \rho)$, with $1 \leq k \leq 7$, depending

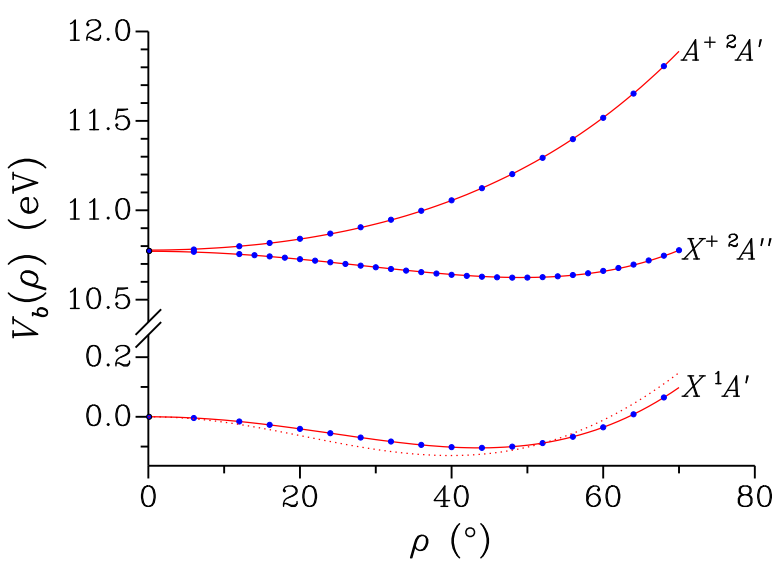

Figure 7. For the neutral species $\tilde{X}^{1} A^{\prime}$ ground state and the cationic species $\tilde{X}^{+2} A^{\prime \prime}$ and $\tilde{A}^{+2} A^{\prime}$ states, variations with $\rho$ of the bending potential $V_{b}(\rho)$ of Eq. (6). Ab initio values are indicated by dots; solid lines are fitted values calculated with Eq. (3). The dotted line is the bending potential retrieved by Koput $^{8}$ for the neutral ground state.

only on the two active coordinates. Such atom positions are obtained using the coordinate system of Section III A and setting the inactive coordinates to the equilibrium values of Section IIIA 2. The molecule fixed $x y z$ axis system, shown in Fig. 2, is such that its origin is the molecular center of mass; its $x z$ plane is parallel to the $\mathrm{C}_{\alpha} \mathrm{NC}$ plane; and the $A B$ axis, bisecting the $\angle \mathrm{C}_{\alpha} \mathrm{NC}$ angle, is parallel to the $x$ axis.

In order to use previous theoretical results ${ }^{37-42}$ concerning large amplitude bending modes, the angular coordinate $\rho$ is replaced by $t=\cos (\pi-\rho)$, where $-1 \leq t \leq$ +1 . The classical rotation-bending-torsion kinetic energy $T$ is expressed in terms of the angular velocities $\omega_{\delta}$, with $\delta=x, y, z$, the time derivatives $\omega_{\tau}=\dot{\tau}$ and $\omega_{t}=\dot{t}$, and the $5 \times 5$ generalized inertia tensor $\mathbf{I}(\tau, t)$ as:

$$
T=\frac{1}{2} \sum_{\delta, \gamma} I(\tau, t)_{\delta \gamma} \omega_{\delta} \omega_{\gamma},
$$

where $\delta, \gamma=x, y, z, \tau, t$. As stressed in Section III A 2, the equilibrium values of the inactive coordinates are only available for $\tau=0$ or $\tau=60^{\circ}$. For this reason the $\tau$ dependence of the generalized inertia tensor in Eq. (7) is ignored and this tensor is approximated by $\mathbf{I}(t)$. This approximated tensor is to be calculated for the $\tau=0$ configuration of the neutral and the cation ground states, and for the $\tau=60^{\circ}$ configuration of the cation $\tilde{A}^{+2} A^{\prime}$ state. The zeroth-order Hamiltonian $H_{\mathrm{rbt}}$, written in terms of the generalized inverse inertia tensor ${ }^{43,44} \boldsymbol{\mu}(t)$, takes the form:

$$
\begin{aligned}
& H_{\mathrm{rbt}}=\frac{1}{2} P_{\tau} \mu_{\tau \tau} P_{\tau}+\frac{1}{2} P_{t} \mu_{t t} P_{t}+\frac{1}{2} \sum_{\delta=x, y, z} \mu_{\delta \delta} J_{\delta}^{2} \\
& +\frac{1}{2} \mu_{x z}\left\{J_{x}, J_{z}\right\}+\frac{1}{2}\left\{\mu_{x \tau}, P_{\tau}\right\} J_{x} \\
& +\frac{1}{2}\left\{\mu_{y t}, P_{t}\right\} J_{y}+\frac{1}{2}\left\{\mu_{z \tau}, P_{\tau}\right\} J_{z}+V_{0}(\tau, t),
\end{aligned}
$$

where $\mu_{\delta \gamma}$ are $t$-dependent components of the general- 
ized inverse inertia tensor; $\{$,$\} is the anticommutator;$ $P_{\tau}$ and $P_{t}$ are the momentum conjugated to $\tau$ and $t$, respectively; $J_{\delta}$, with $\delta=x, y, z$, are molecule fixed components of the rotational angular momentum $\mathbf{J}$; and $V_{0}(\tau, t)$ is the anharmonic potential of Eq. (6). The volume element to be used is $\sin \theta \mathrm{d} \theta \mathrm{d} \phi \mathrm{d} \chi \mathrm{d} \tau \mathrm{d} t$. A term involving the determinant of $\boldsymbol{\mu}(t)$, giving rise to a mass-dependent potential, ${ }^{43,44}$ has been omitted in Eq. (8). Because of the $C_{s}$ symmetry of the molecule, the generalized inverse inertia tensor displays only 4 nonvanishing non-diagonal components. Compared to the rotation-bending-torsion Hamiltonian in Eq. (4) of Koput, ${ }^{8}$ the additional term in $\mu_{y t}$ arising in this work is due to a different molecule fixed axis system.

Although there are no analytical expressions for the generalized inverse inertia tensor, it can be shown that its $\mu_{z z}, \mu_{x \tau}, \mu_{z \tau}$, and $\mu_{\tau \tau}$ components go to infinity for the $t=-1, C_{3 v}$ configuration. This is due to the quasisymmetry. Using Appendix B, it can be deduced that these components and $\mu_{t t}$, display the following limiting behavior when $t \rightarrow-1$ :

$$
\lim _{t \rightarrow-1}\left\{\begin{array}{l}
\mu_{z z}(t)=B / z, \quad \mu_{x \tau}(t)=-2 A / \sqrt{2 z} \\
\mu_{z \tau}(t)=B / z, \quad \mu_{\tau \tau}(t)=B / z \\
\mu_{t t}(t)=4 B z
\end{array}\right.
$$

where $z=1+t$ and the kinetic energy constants $A$ and $B$ can be expressed in terms of atom masses and $C_{3 v}$ configuration geometrical parameters using Eqs. (B3).

In order to compute bending-torsion energies, the zeroth-order Hamiltonian of Eq. (8) is diagonalized using the following basis set functions:

$$
\psi_{m, v}(\tau, t)=|m\rangle \phi_{v}^{m}(t),
$$

where $|m\rangle=\exp (i m \tau) / \sqrt{2 \pi}$ is a free internal rotation function characterized by the quantum number $m$ and $\phi_{v}^{m}(t)$ is a bending function characterized by the quantum number $v$ and depending on $m$. The bending functions are the eigenfunctions of a bending Hamiltonian $H_{\mathrm{b}}^{m}$ built retaining in Eq. (8) those terms having diagonal torsional matrix elements:

$$
H_{\mathrm{b}}^{m}=\frac{1}{2} \mu_{\tau \tau} m^{2}+\frac{1}{2} P_{t} \mu_{t t} P_{t}+V_{\mathrm{b}}(t),
$$

where $V_{\mathrm{b}}(t)$ is the pure bending potential of Eq. (6). The results of Eq. (9) suggest that the bending eigenfunctions and eigenvalues should be obtained using the results derived for a large amplitude bending mode. ${ }^{37-42}$ We are led to expand the bending eigenfunction $\phi_{v}^{m}(t)$ as:

$$
\phi_{v}^{m}(t)=\sum_{p=0}^{p_{\mathrm{Max}}} a_{p}^{v, m} \theta_{p}^{\alpha, \beta}(t),
$$

where $a_{p}^{v, m}$ are expansion coefficients and $\theta_{p}^{\alpha, \beta}(t)$ are the basis set functions defined in Eq. (4) of Coudert. ${ }^{38}$ In agreement with Eqs. (9), $\beta$ is set to $|m|$. As the bending potentials, shown in Fig. 7 , become strongly repulsive when $t$ increases, the bending functions should vanish in this limiting case. This behavior is achieved by setting $\alpha$ to a large enough positive value. Taking $\alpha=30$ ensures the correct behavior without accuracy loss. The matrix of the bending Hamiltonian $H_{\mathrm{b}}^{m}$ can be set up evaluating the matrix elements of the bending potential $V_{\mathrm{b}}(t)$ with a Gauss-Jacobi quadrature. Matrix elements of the operators $P_{t} \mu_{t t} P_{t}$ and $\mu_{\tau \tau}$ can be evaluated with the help of Eqs. (A1)-(A6) of Coudert et al. ${ }^{45}$ The bending energies obtained after diagonalization will be denoted $E_{\mathrm{b}}^{m}(v)$. The matrix element of the zeroth order Hamiltonian in Eq. (8) can now be expressed as:

$$
\begin{aligned}
& \left\langle\psi_{m^{\prime}, v^{\prime}}\left|H_{\mathrm{rbt}}\right| \psi_{m, v}\right\rangle=\delta_{m^{\prime}, m} \delta_{v^{\prime}, v} E_{\mathrm{b}}^{m}(v) \\
& -\frac{1}{4} V_{3}^{0} \delta_{\left|m^{\prime}-m\right|, 3}\left\langle\phi_{v^{\prime}}^{m^{\prime}}|\rho| \phi_{v}^{m}\right\rangle,
\end{aligned}
$$

where the last term, when evaluated with the help of Eq. (12), leads to the bending matrix element $\left\langle\theta_{p^{\prime}}^{\alpha^{\prime}, \beta^{\prime}}|\rho| \theta_{p}^{\alpha, \beta}\right\rangle$. Evaluation of this matrix element should be carried out with care because the angle $\rho$ cannot be expanded as a polynomial-type expansion in terms of $t$. The angle $\rho$ varies like $\sqrt{1-t^{2}}$ when $t$ is close to -1 and its bending matrix element can be computed using the same ideas as in Eqs. (A1)-(A6) of Coudert et al. ${ }^{45}$ Equation (13) emphasizes that the matrix of the zeroth order Hamiltonian can be split into 3 submatrices. The matrices with $m=3 q \pm 1$ give rise to doubly degenerate levels belonging to the $E$ symmetry species of $C_{3 v}$. The submatrix with $m=3 q$ can be further split into 2 smaller submatrices leading to nondegenerate levels belonging to either the $A_{1}$ or $A_{2}$ symmetry species. Bending-torsion energy levels obtained after diagonalization of the zeroth order Hamiltonian are denoted $E\left(v_{b}, m, \Gamma\right)$ and labeled using the bending vibrational quantum number $v_{b}$, the torsional quantum number $m$, and $\Gamma$ their symmetry species in $C_{3 v}$. The bending-torsion wavefunction takes the following form:

$$
\left|\Psi_{v_{b}, m, \Gamma}\right\rangle=\sum_{q, v} b_{q, v}^{v_{b}, m, \Gamma}\left|\psi_{q, v}\right\rangle,
$$

where $b_{q, v}^{v_{b}, m, \Gamma}$ are expansion coefficients and $\psi_{q, v}$ is a basis function of Eq. (10). Table III gathers four sets of calculated bending-torsion energies listed with respect to that of the lowest $v_{b}=m=0, A_{1}$ level. The first set was calculated using the reference configuration and the anharmonic potential of Koput, ${ }^{8}$ defined in Column I of his Table III. The energy values are at most within $0.07 \mathrm{~cm}^{-1}$ from those reported by the author in his Table II. The second, third, and fourth sets, for the neutral molecule and the cationic species $\tilde{X}^{+2} A^{\prime \prime}$ and $\tilde{A}^{+2} A^{\prime}$ states, respectively, were calculated using the reference configurations obtained in Section III A 2 and the anharmonic potential of Eq. (6). For the neutral molecule and especially for $v_{b}=1$, the energy values obtained in this work differ from those obtained with Koput's parameterization ${ }^{8}$ because, as emphasized by Fig. 7, the bending potentials are very different. Calculating the 
Table III. Calculated bending-torsion energies ${ }^{\mathrm{a}}$

\begin{tabular}{rrrrrrr}
\hline \hline & & & \multicolumn{2}{c}{$\tilde{X}^{1} A^{\prime}$} & $\tilde{X}^{+2} A^{\prime \prime}$ & $\tilde{A}^{+2} A^{\prime}$ \\
\cline { 4 - 5 }$v_{b}$ & $m$ & $\Gamma$ & Ref. $^{8}$ & This work & & \\
\hline 0 & 1 & $E$ & 8.70 & 8.69 & 6.63 & 190.57 \\
0 & -2 & $E$ & 36.32 & 36.52 & 36.00 & 388.36 \\
0 & 3 & $A_{2}$ & 80.16 & 80.30 & 72.97 & 593.57 \\
0 & 3 & $A_{1}$ & 80.45 & 80.64 & 74.93 & 593.81 \\
0 & 4 & $E$ & 141.71 & 141.77 & 128.71 & 808.33 \\
1 & 0 & $A_{1}$ & 191.28 & 168.34 & 183.06 & 339.90 \\
1 & 1 & $E$ & 200.74 & 177.98 & 190.32 & 534.12 \\
\hline \hline
\end{tabular}

${ }^{a}$ Bending-torsion levels are labeled with the quantum numbers $v_{b}$ and $m$, and their symmetry species $\Gamma$. Their energies in $\mathrm{cm}^{-1}$ are given with respect to the $v_{b}=m=0, A_{1}$ lowest level. The reference configuration of Koput ${ }^{8}$ and those retrieved in Section III A 2 for the neutral and the cationic species were considered.

fundamental frequency of the bending $\angle \mathrm{C}_{\alpha} \mathrm{NC}$ mode for the neutral species as the average of the energy differences $E\left(v_{b}=1, m=1, E\right)-E\left(v_{b}=0, m=1, E\right)$ and $E\left(v_{b}=1, m=0, A_{1}\right)-E\left(v_{b}=0, m=0, A_{1}\right)$, we obtain 191.6 and $168.8 \mathrm{~cm}^{-1}$ with Koput's parameterization ${ }^{8}$ and that of this work, respectively. Both values compare favorably with the experimental gas-phase value of $172 \mathrm{~cm}^{-1}$ reported by Sullivan et al. ${ }^{19}$ Similarly, also for the neutral, the fundamental frequency for the torsional mode should be calculated as the average of the energy difference $E\left(v_{b}=0, m=3, A_{2}\right)-E\left(v_{b}=0, m=0, A_{1}\right)$ and $E\left(v_{b}=0, m=-2, E\right)-E\left(v_{b}=0, m=1, E\right)$ leading to $54 \mathrm{~cm}^{-1}$ with either Koput's parameterization ${ }^{8}$ or that of this work. This value is in very good agreement with the experimental gas-phase value reported by Sullivan et al. ${ }^{19} 50 \mathrm{~cm}^{-1}$.

\section{Vibration-bending-torsion energy levels}

Vibration-bending-torsion energy levels were calculated using the harmonic adiabatic approximation ${ }^{23}$ (HADA). Eigenvalues and eigenfunctions of the total Hamiltonian are retrieved using basis set functions which are the product of the harmonic oscillator wavefunction of Eq. (5) and of the bending-torsion basis set functions of Eq. (10):

$$
\Psi_{\mathbf{n}}(\mathbf{Q} ; t) \psi_{m, v}(\tau, t)
$$

Within the HADA approximation, ${ }^{23}$ the $t$ parametric dependence of the harmonic oscillator wavefunction is ignored leading to no additional kinetic energy term in the zeroth-order Hamiltonian of Eq. (8). Also, matrix elements of the total Hamiltonian are only considered between two basis set functions of Eq. (15) characterized by the same $n_{1}, \ldots, n_{5}$ vibrational quantum numbers. We are led to compute bending-torsion energy levels for each
Table IV. Calculated vibration-bending-torsion energies ${ }^{\mathrm{a}}$

\begin{tabular}{ccccrrr}
\hline \hline $\mathbf{n}$ & $v_{b}$ & $m$ & $\Gamma$ & $\tilde{X}^{1} A^{\prime}$ & $\tilde{X}^{+2} A^{\prime \prime}$ & $\tilde{A}^{+2} A^{\prime}$ \\
\hline Ground & 0 & 1 & $E$ & 8.69 & 6.63 & 190.57 \\
$\nu_{1}$ & 0 & 0 & $A_{1}$ & 2336.94 & 2104.05 & 2010.75 \\
& 0 & 1 & $E$ & 2345.31 & 2110.91 & 2203.06 \\
$\nu_{2}$ & 0 & 0 & $A_{1}$ & 1483.62 & 1338.55 & 1518.59 \\
& 0 & 1 & $E$ & 1492.00 & 1344.74 & 1707.25 \\
$\nu_{3}$ & 0 & 0 & $A_{1}$ & 990.17 & 920.18 & 753.84 \\
& 0 & 1 & $E$ & 999.15 & 927.02 & 950.18 \\
$\nu_{4}$ & 0 & 0 & $A_{1}$ & 620.92 & 540.84 & 551.92 \\
& 0 & 1 & $E$ & 629.51 & 547.42 & 740.52 \\
$\nu_{5}$ & 0 & 0 & $A_{1}$ & 587.78 & 455.54 & 583.77 \\
& 0 & 1 & $E$ & 596.51 & 462.30 & 782.97 \\
\hline \hline
\end{tabular}

a Vibration-bending-rotation levels are labeled with the small amplitude vibrational quantum number $\mathbf{n}$, the bending quantum numbers $v_{b}$, the torsional quantum number $m$, and their torsional symmetry species $\Gamma$. Their energies in $\mathrm{cm}^{-1}$ with respect to the $v_{b}=m=0, A_{1}$ ground vibrational state are listed in the body of the table.

small amplitude vibrational state using the results in Section III B and adding the following term to the potential energy function:

$$
V_{\mathbf{n}}(t)=\sum_{i=1}^{5} \tilde{\nu}_{i}(t) n_{i}
$$

where $\mathbf{n}=n_{1}, \ldots, n_{5}$, as in Eq. (5), and $n_{i}$ appears, instead of the familiar $n_{i}+\frac{1}{2}$, because the zero-point vibrational energy is already included in the anharmonic potential of Eq. (6)

Table IV lists a few low-lying vibration-bendingtorsion energies for the neutral and the cationic species. It can be seen that for the ground vibrational state of either electronic states, the energies are the same as in Table III. This is due to the fact that the contribution from the potential in Eq. (16) vanishes for that state. An estimate of the fundamental frequencies of the 5 small amplitude vibrational modes can be obtained taking the average of the energy differences $E\left(\nu_{i}, v_{b}=\right.$ $\left.0, m=0, A_{1}\right)-E\left(\right.$ Ground, $\left.v_{b}=0, m=0, A_{1}\right)$ and $E\left(\nu_{i}, v_{b}=0, m=1, E\right)-E\left(\right.$ Ground, $\left.v_{b}=0, m=1, E\right)$. For the neutral molecule this leads to 2337, 1483, 990, 621 , and $588 \mathrm{~cm}^{-1}$. These frequencies should be compared to the experimental values of Sullivan et al., ${ }^{19}$ $2230,1434,855,619$, and $583 \mathrm{~cm}^{-1}$. In many cases, the frequencies retrieved in this work are in better agreement with the experimental values than those calculated by these authors. For the cation $\tilde{X}^{+2} A^{\prime \prime}$ state, the fundamental frequencies are 2104, 1338, 920, 541, and $456 \mathrm{~cm}^{-1}$ and, as stressed in Section II, are consistent with the observed values extracted from the experimental TPES. 


\section{CALCULATED TPES}

For a transition with upper and lower levels denoted $v^{+}$and $v^{\prime \prime}$, respectively, belonging to either ionizing transitions, the transition probability is taken equal to the Franck-Condon factor $\left|\left\langle\Psi_{v^{+}} \mid \Psi_{v^{\prime \prime}}\right\rangle\right|^{2}$ involving $\Psi_{v^{+}}$the wavefunction of the cation and $\Psi_{v^{\prime \prime}}$ that of the neutral.

\section{A. Bending-torsion approach}

The expansion of the bending-torsion wavefunction in Eq. (14) shows that computing Franck-Condon factors requires evaluating the matrix elements of two basis set functions of Eq. (10):

$$
\left\langle\psi_{m^{+}, v^{+}} \mid \psi_{m^{\prime \prime}, v^{\prime \prime}}\right\rangle=\delta_{m^{+}, m^{\prime \prime}}\left\langle\phi_{v^{+}}^{m^{+}} \mid \phi_{v^{\prime \prime}}^{m^{\prime \prime}}\right\rangle
$$

The last term in this equation can be obtained from Eq. (12) leading to bending matrix elements of the form $\left\langle\theta_{p^{+}}^{\alpha^{+}, \beta^{+}} \mid \theta_{p^{\prime \prime}}^{\alpha^{\prime \prime}, \beta^{\prime \prime}}\right\rangle$. The latter reduces to $\delta_{p^{+}, p^{\prime \prime}}$ because $m^{+}=m^{\prime \prime}$ in Eq. (17) dictates that $\alpha^{+}=\alpha^{\prime \prime}$ and $\beta^{+}=\beta^{\prime \prime}$, in agreement with Section III B. For each electronic state, the lowest 81 bending-torsion energy levels were calculated for the $A_{1}$ and $A_{2}$ torsional symmetry species as well as for the $E$ torsional symmetry species. Franck-Condon factors were then computed for the resulting transitions of either ionizing transitions. Symmetry requires that transitions connect bending-torsion energy levels with the same torsional symmetry, reducing the number of allowed transitions. The TPES was calculated for both ionizing transitions and for a temperature of $300 \mathrm{~K}$. The value of the adiabatic ionization energy for the $\tilde{X}^{+2} A^{\prime \prime} \leftarrow \tilde{X}^{1} A^{\prime}$ ionizing transition calculated at $10.729 \mathrm{eV}$ was found too high when first comparing observed and calculated TPES. Matching the position of the strongest peaks, this value was decreased by $0.133 \mathrm{eV}$ yielding $10.596(6) \mathrm{eV}$. As stressed in Section II B, no spectroscopic feature could be assigned for the $\tilde{A}^{+2} A^{\prime} \leftarrow \tilde{X}^{1} A^{\prime}$ ionizing transition. For this reason, the adiabatic ionization energy of this transition, calculated at $10.892 \mathrm{eV}$, was also decreased by $0.133 \mathrm{eV}$ yielding $10.759(50) \mathrm{eV}$ where the large $50 \mathrm{meV}$ uncertainty is an estimate of the $\tilde{A}^{+2} A^{\prime}$ and $\tilde{X}^{+2} A^{\prime \prime}$ states relative energy $a b$ initio error. Figure 8 depicts the resulting TPES along with the experimental one. For the $\tilde{X}^{+2} A^{\prime \prime} \leftarrow \tilde{X}^{1} A^{\prime}$ ionizing transition, only 4 sharp peaks arise and the short Franck-Condon progression is due to the bending mode. For the $\tilde{A}^{+2} A^{\prime} \leftarrow \tilde{X}^{1} A^{\prime}$ ionizing transition, the larger number of peaks is due to the large difference between the structure and the potential of the neutral and of the cation $\tilde{A}^{+2} A^{\prime}$ state. The TPES including both ionizing transitions was computed multiplying the contribution from the $\tilde{A}^{+2} A^{\prime} \leftarrow \tilde{X}^{1} A^{\prime}$ ionizing transition by 3 which ensures the best agreement with the experimental spectrum but has no theoretical justification. An HWHM of $75 \mathrm{~cm}^{-1}$ was used to take into account

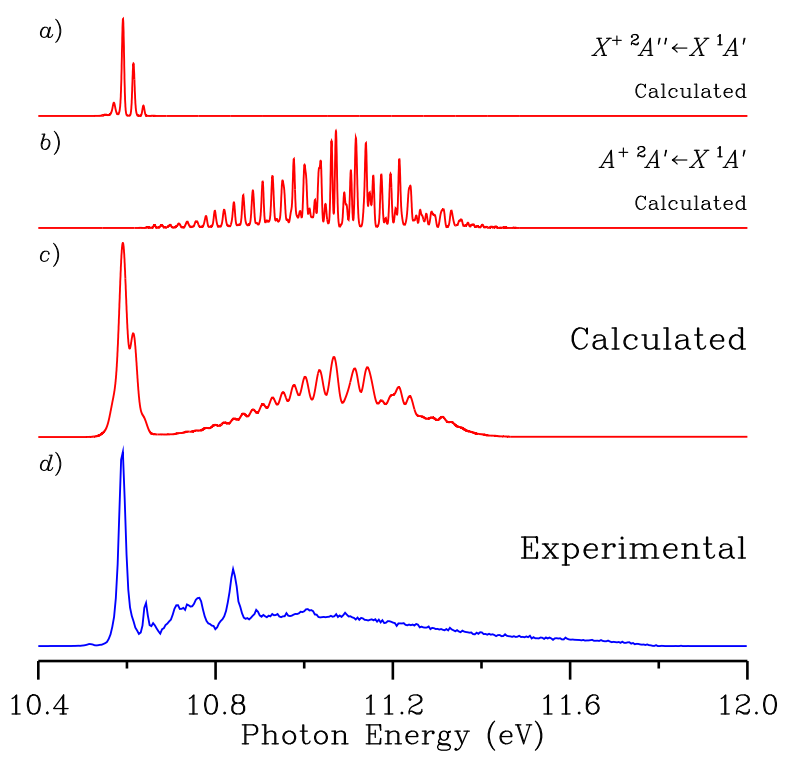

Figure 8. TPES calculated with the bending-torsion approach plotted as a function of the photon energy. A Gaussian line shape with an HWHM of $20 \mathrm{~cm}^{-1}$ was taken for clarity in a) and $b$ ) for the $\tilde{X}^{+2} A^{\prime \prime} \leftarrow \tilde{X}^{1} A^{\prime}$ and $\tilde{A}^{+2} A^{\prime} \leftarrow \tilde{X}^{1} A^{\prime}$ ionizing transitions, respectively. In $c$ ), an HWHM of $75 \mathrm{~cm}^{-1}$ was adopted for the TPES calculated in accordance with Section IV A and compared to the experimental spectrum in $d$ ). Different $y$-axis scales were used for each panel.

both the experimental resolution and the rotational envelope. Although the agreement with the experimental TPES is not really satisfactory, the present simplified calculation is able to reproduce the broad feature spanning the energy range from 10.6 to $11.8 \mathrm{eV}$.

\section{B. Vibration-bending-torsion approach}

Using the results in Section III C we can see that evaluating the Franck-Condon factors leads to matrix elements between two basis set functions of Eq. (15) expressed as:

$$
\left\langle\psi_{m^{+}, v^{+}}\left|I\left(\mathbf{n}^{+}, \mathbf{n}^{\prime \prime} ; t\right)\right| \psi_{m^{\prime \prime}, v^{\prime \prime}}\right\rangle
$$

where $I\left(\mathbf{n}^{+}, \mathbf{n}^{\prime \prime} ; t\right)$ is the Franck-Condon overlap integral between $\Psi_{\mathbf{n}^{+}}\left(\mathbf{Q}^{+} ; t\right)$ the vibrational wavefunctions of the cation and $\Psi_{\mathbf{n}^{\prime \prime}}\left(\mathbf{Q}^{\prime \prime} ; t\right)$ that of the neutral, as defined in Eq. (5). The Franck-Condon overlap integral takes the form: ${ }^{46}$

$$
\begin{aligned}
& I\left(\mathbf{n}^{+}, \mathbf{n}^{\prime \prime} ; t\right)=[\operatorname{det} \mathbf{J}(t)]^{-\frac{1}{2}} \\
& \quad \times \int \Psi_{\mathbf{n}^{+}}\left(\mathbf{Q}^{+} ; t\right) \Psi_{\mathbf{n}^{\prime \prime}}\left(\mathbf{Q}^{\prime \prime} ; t\right) d Q^{\prime \prime},
\end{aligned}
$$

where $\mathbf{J}(t)$ is defined below. The relation between both sets of normal coordinates, required to evaluate this integral, is Duschinsky's linear transformation: ${ }^{47}$

$$
\mathbf{Q}^{+}=\mathbf{J}(t) \mathbf{Q}^{\prime \prime}+\mathbf{k}(t),
$$


where $\mathbf{J}(t)$ is a matrix and $\mathbf{k}(t)$ is a column vector. Denoting $\mathbf{L}^{+}(t)$ and $\mathbf{L}^{\prime \prime}(t)$ the Wilson-type matrices ${ }^{34}$ arising in Section III A 3 for the cation and the neutral species, respectively, we obtain: ${ }^{48}$

$$
\mathbf{J}(t)=\left[\mathbf{L}^{+}(t)\right]^{-1} \mathbf{L}^{\prime \prime}(t)
$$

and

$$
\mathbf{k}(t)=\left[\mathbf{L}^{+}(t)\right]^{-1}\left[\mathbf{q}^{\mathrm{eq}^{\prime \prime}}(t)-\mathbf{q}^{\mathrm{eq}^{+}}(t)\right]
$$

where $\mathbf{q}^{\mathrm{eq}}(t)$ and $\mathbf{q}^{\mathrm{eq}^{\prime \prime}}(t)$ are the equilibrium values arising in Section III A 2 for the cation and the neutral species, respectively. Using Ruhoff and Ratner's recursive algorithm ${ }^{46}$ and Eqs. (20)-(22), the Franck-Condon overlap integral were evaluated. For each set $\mathbf{n}^{+}$and $\mathbf{n}^{\prime \prime}$ of upper and lower vibrational quantum numbers, a $t$-dependent function was obtained. Figure 9 shows the variations of $I\left(\mathbf{n}^{+}, \mathbf{n}^{\prime \prime} ; t\right)$ for the $\tilde{A}^{+2} A^{\prime} \leftarrow \tilde{X}^{1} A^{\prime}$ ionizing transition and for 4 sets of vibrational quantum numbers. The cation, $\mathbf{n}^{+}=\nu_{n}$ was taken, with $1 \leq n \leq 4$; while for the neutral, $\mathbf{n}^{\prime \prime}$ corresponds to the ground vibrational state.

Because the Franck-Condon overlap integral is not $\tau$ dependent, the matrix element in Eq. (18) reduces to:

$$
\delta_{m^{+}, m^{\prime \prime}}\left\langle\phi_{v^{+}}^{m^{+}}\left|I\left(\mathbf{n}^{+}, \mathbf{n}^{\prime \prime} ; t\right)\right| \phi_{v^{\prime \prime}}^{m^{\prime \prime}}\right\rangle,
$$

an expression analogous to that in Eq. (17). As in the case of this equation, the following bending matrix element arises:

$$
\left\langle\theta_{p^{+}}^{\alpha, \beta}\left|I\left(\mathbf{n}^{+}, \mathbf{n}^{\prime \prime} ; t\right)\right| \theta_{p^{\prime \prime}}^{\alpha, \beta}\right\rangle,
$$

and can be calculated using a Gauss-Jacobi quadrature.

The TPES of each ionizing transition was simulated considering small amplitude vibrational states with $\sum_{i=1}^{5} n_{i}^{\prime \prime} \leq 2$ for the neutral species and $\sum_{i=1}^{5} n_{i}^{+} \leq 4$ for the cation leading to 21 and 126 states, respectively. For each state 162 bending-torsion energy levels were calculated including 81 levels with $A_{1}$ and $A_{2}$ torsional symmetry and 81 levels with $E$ torsional symmetry. This led to 3402 energy levels for the neutral molecule and 20412 for the cationic species. Total photoionization probabilities were then computed for the resulting transitions taking into account symmetry restrictions.

In addition to the experimental TPES, Fig. 10 shows three TPES calculated for a vibrational temperature of $300 \mathrm{~K}$ including those of the $\tilde{X}^{+2} A^{\prime \prime} \leftarrow \tilde{X}^{1} A^{\prime}$ and $\tilde{A}^{+2} A^{\prime} \leftarrow \tilde{X}^{1} A^{\prime}$ ionizing transitions, and a trial TPES computed adding the contribution of both ionizing transitions. In order to have the best agreement with the experimental TPES, the contribution from the $\tilde{A}^{+2} A^{\prime} \leftarrow$ $\tilde{X}^{1} A^{\prime}$ ionizing transition was multiplied by 2 . This factor, as the one used in Section IV A, has no theoretical significance. Comparing this trial TPES with the experimental one confirms the line position agreement highlighted in Section II. It also shows that the broad feature of the experimental TPES, from 10.6 to $11.8 \mathrm{eV}$,

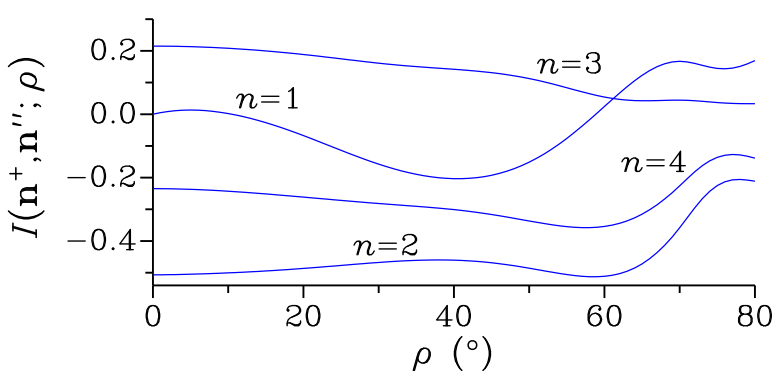

Figure 9. The dimensionless Franck-Condon overlap integral $I\left(\mathbf{n}^{+}, \mathbf{n}^{\prime \prime} ; \rho\right)$ for the $\tilde{A}^{+2} A^{\prime} \leftarrow \tilde{X}^{1} A^{\prime}$ ionizing transition is plotted as a function of $\rho$ for 4 sets of small amplitude vibrational states. $\mathbf{n}^{+}=\nu_{n}$, with $1 \leq n \leq 4$, and $\mathbf{n}^{\prime \prime}$ corresponding to the neutral ground vibrational state. The value of $n$ identifies each curve in the figure.

is fairly well reproduced. The line intensity agreement is, however, less satisfactory. The sharp peaks in the 10.65 to $10.8 \mathrm{eV}$ energy range, corresponding to the $\nu_{2}$, $\nu_{3}$, and $\nu_{4}$ modes, are obviously too weak. Conversely, the Franck-Condon progression due to the $\angle \mathrm{C}_{\alpha} \mathrm{NC}$ bending mode at $191 \mathrm{~cm}^{-1}$ is quite prominent in the calculated TPES but not in the experimental one. Without adjustment, the calculated adiabatic ionization energies for the $\tilde{X}^{+2} A^{\prime \prime} \leftarrow \tilde{X}^{1} A^{\prime}$ and $\tilde{A}^{+2} A^{\prime} \leftarrow \tilde{X}^{1} A^{\prime}$ ionizing transitions are exactly the same as in Section IV A. In this case too, the value for the former transition was decreased being too large. Within the experimental uncertainty, the energy shift was the same as in this section leading to an adjusted value of $10.596(6) \mathrm{eV}$. Similarly for the $\tilde{A}^{+2} A^{\prime} \leftarrow \tilde{X}^{1} A^{\prime}$ ionizing transition, this leads to the same estimated value of $10.759(50) \mathrm{eV}$.

Although line intensities may be affected by resonant autoionization in threshold photoelectron spectroscopy, ${ }^{49}$ the intensity discrepancies in the 10.65 to $10.8 \mathrm{eV}$ energy range can also be understood noticing that the FranckCondon factors are extremely sensitive to the equilibrium value differences in Eq. (22). The $\tilde{X}^{+2} A^{\prime \prime} \leftarrow \tilde{X}^{1} A^{\prime}$ ionizing transition was recalculated changing slightly the parameters retrieved whilst fitting the $a b$ initio values of the cationic species $\tilde{X}^{+2} A^{\prime \prime}$ ground state in Section III A 2. For the two bond length coordinates $q_{3}=r(\mathrm{CN})$ and $q_{4}=r(\mathrm{CO})$, the values of $q_{3}^{0}$ and $q_{4}^{0}$ in Eq. (3) were increased by 1.721 and $0.904 \%$, respectively. For the bond angle $q_{6}=\angle \mathrm{NCO}$, the parameters $f_{j}^{6}$ in Eq. (4) were increased by $20 \%$. The vibration-bending-torsion energies of the cationic species $\tilde{X}^{+2} A^{\prime \prime}$ ground state underwent small changes on the order of a few $\mathrm{cm}^{-1}$ but line intensities were drastically changed. Figure 11 shows the TPES obtained adding the contribution of both ionizing transitions with no multiplicative factors. The FranckCondon progressions due to the $\nu_{1}$ and $\nu_{2}$ modes and the fundamentals of the $\nu_{3}$ and $\nu_{4}$ modes can now clearly be seen in panel $a$ ) of this figure where vibrational assignments are given. These assignments are consistent with Table I and a more satisfactory agreement with the experimental TPES arises. However, we are still un- 


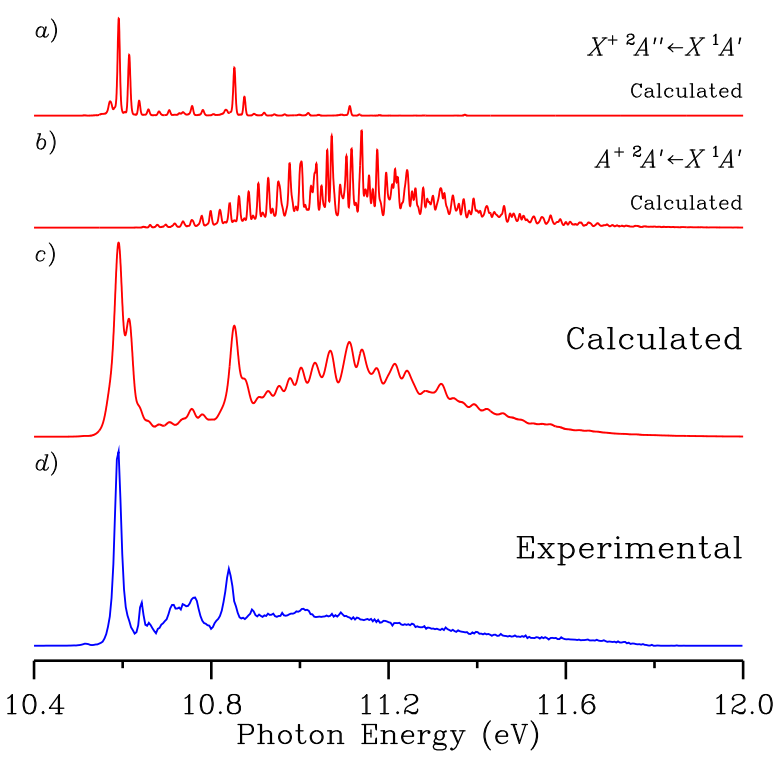

Figure 10. TPES calculated with the vibration-bendingtorsion approach plotted as a function of the photon energy. The $\tilde{X}^{+2} A^{\prime \prime} \leftarrow \tilde{X}^{1} A^{\prime}$ and $\tilde{A}^{+2} A^{\prime} \leftarrow \tilde{X}^{1} A^{\prime}$ ionizing transitions are drawn in $a$ ) and $b$ ), respectively, using the same line shape as for $a$ ) in Fig. 8. The TPES calculated in accordance with Section IV B is plotted in $c$ ) using the same line shape as for $c$ ) in Fig. 8 and compared to the experimental spectrum in $d$ ). Different $y$-axis scales were used for each panel.

able to assign peak \# 3. Likewise, the band calculated at $10.61 \mathrm{eV}$, corresponding to the short Franck-Condon progression along the bending molde as described in Section IV A, still does not have a counterpart in the experimental TPES. No effort was made to recalculate the $\tilde{A}^{+2} A^{\prime} \leftarrow \tilde{X}^{1} A^{\prime}$ ionizing transition. This transition is dominated by Franck-Condon progressions due to the two large amplitude modes and altering the parameterization of the small amplitude vibrational modes did not allow us to observe Franck-Condon progressions due to these modes.

\section{CONCLUSION}

The photoelectron spectrum of methyl isocyanate in the 10.4 to $12 \mathrm{eV}$ region is attributable to ionization from the nonbonding $\pi_{2}\left(a^{\prime \prime}, a^{\prime}\right)$ orbitals of the $\mathrm{NCO}$ group leading to two ionizing transitions from the neutral ground $\tilde{X}^{1} A^{\prime}$ state to the cation ground $\tilde{X}^{+2} A^{\prime \prime}$ and first excited $\tilde{A}^{+2} A^{\prime}$ electronic states. The TPES of these two photoionizing transitions has been recorded in this work using synchrotron radiation and theoretically modeled.

Although methyl isocyanate has only 7 atoms, it is theoretically challenging as it displays two large amplitude motions consistent with its quasi-symmetry. ${ }^{2-11}$ The coupling between these two large amplitude motions must be treated exactly to properly model its energy level dia-

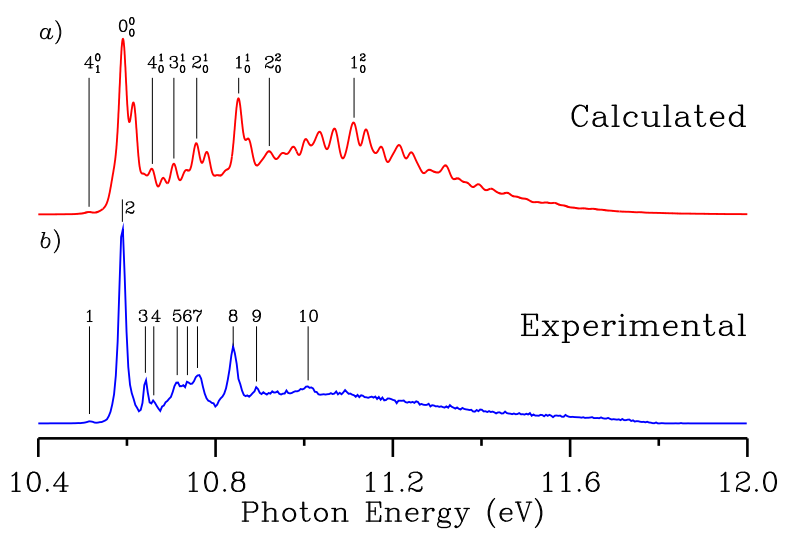

Figure 11. TPES calculated with the vibration-bendingtorsion approach plotted in $a$ ) as a function of the photon energy. The line shape is the same as for $c$ ) in Fig. 8. This adjusted spectrum was obtained by changing slightly parameters describing the equilibrium values of the inactive coordinates $q_{3}, q_{4}$, and $q_{6}$ for the cationic species $\tilde{X}^{+2} A^{\prime \prime}$ ground state. The vibrational bands of the $\tilde{X}^{+2} A^{\prime \prime} \leftarrow \tilde{X}^{1} A^{\prime}$ ionizing transition are labeled with the small amplitude vibrational mode number of Section III C and upper and lower vibrational quantum numbers. The experimental spectrum is shown in $b$ ) and the 10 strongest sharp peaks are numbered as in Fig. 1.

gram and, for instance, to account for its high-resolution spectroscopic data. ${ }^{8,13,14}$ As the TPES recorded in this work displays well-resolved vibrational structures, the 13 small amplitude vibrational modes should also be included in the theoretical model.

Even though theoretical models accounting for one large amplitude motion have already been proposed for modeling photoelectronic spectra, ${ }^{50}$ the model developed in this work goes one step further since it takes into account two large amplitude motions and the small amplitude vibrational modes. Several approximations, however, were made. Only a few small amplitude modes were considered. The selected ones are those closely related to the change in equilibrium structure upon ionization. This principle, applied by Eland ${ }^{25}$ in the case of the parent species HNCO, means in the case of methyl isocyanate that only the 5 small amplitude vibrational modes involving NCO group atoms should be retained. Indeed, the orbital from which ionization occurs belongs to this group and, as confirmed by Table II, the internal coordinates involving these atoms undergo the largest changes when going from the neutral molecule to either states of the cationic species. The second adopted approximation involves neglecting the dependence on the large amplitude torsional motion of the generalized inverse inertia tensor $^{43,44}$ and of the matrices involved in the $F G$ method. ${ }^{34}$ The third approximation involves omitting the vibronic couplings between the two electronic states of the cationic species.

In this work, $a b$ initio calculations were performed to obtain the equilibrium geometries of the neutral and cationic species, the equilibrium values of the small am- 
plitude inactive coordinates as functions of the two active large amplitude coordinates, ${ }^{23,24}$ and the Hessian matrix. These results allowed us to obtain bendingtorsion and vibration-bending-torsion energy levels for the neutral and the cationic species. For the neutral molecule, these results, given in Tables III and IV are in good agreement with those obtained in previous spectroscopic investigations. ${ }^{8,19}$ For the cation $\tilde{X}^{+2} A^{\prime \prime}$ ground electronic state, the vibrational energies could only be compared to those reported by Pasinszki et al. ${ }^{22}$ and those extracted from the present experimental TPES. Table I, where experimental and calculated values are compared, emphasizes that the discrepancies are smaller than $100 \mathrm{~cm}^{-1}$ for fundamental frequencies.

The main results of the paper are the calculated TPES presented in Sections IV A and IV B. With the model in which only the two large amplitude coordinates are considered, a qualitative agreement with the experimental spectrum is achieved as the theoretical TPES displays a sharp line and a broad feature spanning a large energy range, Fig. 8. With the more sophisticated treatment accounting also for the small amplitude vibrational modes, there is a good agreement between the line positions of the strongest sharp peaks, but their intensities are not fully reproduced by the model. A better agreement could be obtained for the line intensity of these peaks by adjusting several parameters retrieved from the fit of the $a b$ initio calculations results.

The present investigation provided an accurate value of $10.596(6) \mathrm{eV}$ for the adiabatic ionization energy of the $\tilde{X}^{+2} A^{\prime \prime} \leftarrow \tilde{X}^{1} A^{\prime}$ photoionizing transition, with either the bending-torsion or the vibration-bending-torsion models. The value obtained directly from the $a b$ initio calculations turned out to be $0.133 \mathrm{eV}$ too high. Although no spectroscopic patterns could be assigned for the $\tilde{A}^{+2} A^{\prime} \leftarrow \tilde{X}^{1} A^{\prime}$ ionizing transition, a value of $10.759(50) \mathrm{eV}$ was deduced for its adiabatic ionization energy using the relative energy of the $\tilde{A}^{+2} A^{\prime}$ and $\tilde{X}^{+2} A^{\prime \prime}$ electronic states obtained from the $a b$ initio calculations. The present investigation also offers an estimate of the oscillator strength ratio between the $\tilde{A}^{+2} A^{\prime} \leftarrow \tilde{X}^{1} A^{\prime}$ and $\tilde{X}^{+2} A^{\prime \prime} \leftarrow \tilde{X}^{1} A^{\prime}$ ionizing transitions. Depending on the model, the value ranges from 1 to 3 .

\section{ACKNOWLEDGMENTS}

This work was performed on the DESIRS beamline under Proposal No. 20160902. We acknowledge SOLEIL for provision of synchrotron radiation facilities and the DESIRS beamline staff for their assistance. This study was supported by the Programme National Physique et Chimie du Milieu Interstellaire (PCMI) of CNRS/INSU with INC/INP co-funded by CEA and CNES. J.-C. G. thanks the CNES for a grant.

\section{DATA AVAILABILITY}

The data that support the findings of this study are available from the corresponding author upon reasonable request.

\section{Appendix A: Fitting expansion to obtain $q_{i}^{\mathrm{eq}}(\tau, \rho)$}

The value of $q_{i}^{0}$ to be used in Eq. (2) are:

$$
q_{i}^{0}= \begin{cases}r_{i}^{0}, & 3 \leq i \leq 5 \\ 0 \text { or } \pi, & 6 \leq i \leq 7 \\ r\left(\mathrm{C}_{\alpha} \mathrm{H}\right), & 8 \leq i \leq 10 \\ \angle \mathrm{NC}_{\alpha} \mathrm{H}, & 11 \leq i \leq 13 \\ 0, & i=14 \\ \pm 2 \pi / 3, & i=15\end{cases}
$$

where $r_{i}^{0}, r\left(\mathrm{C}_{\alpha} \mathrm{H}\right)$, and $\angle \mathrm{NC}_{\alpha} \mathrm{H}$ are the bond lengths and bond angles for the $C_{3 v}$ configuration. The Fouriertype expansions $g_{j}^{i}(\tau)$ in Eq. (2) are given below and are written with parameters denoted $f_{k}^{i, j}$. For $3 \leq i \leq 6$, the expansion $g_{j}^{i}(\tau)$ is given by:

$$
g_{j}^{i}(\tau)=\sum_{k=0}^{m} f_{k}^{i, j} \cos 3(2 k+l) \tau,
$$

where $f_{k}^{i, j}$ are fitted parameters and $l$ is 0 or 1 depending on the coordinate $i$ and on the parity of $j$. For $3 \leq i \leq 5$, $l$ is 0 (1) for $j$ even (odd); for $i=6, l$ is 1 (0) for $j$ even (odd). For $i=7, g_{j}^{i}(\tau)$ is given by:

$$
g_{j}^{i}(\tau)=\sum_{k=0}^{m} f_{k}^{i, j} \sin 3(2 k+l) \tau,
$$

where $l$ is 0 (1) for $j$ even (odd). For the three bond lengths with $8 \leq i \leq 10$ and the three bond angles with $11 \leq i \leq 13, g_{j}^{i}(\tau)$ is given by:

$$
g_{j}^{i}(\tau)=\sum_{k=0}^{m} f_{k}^{i, j} \cos \left[(2 k+l)\left(\tau-l_{i} 4 \pi / 3\right)\right],
$$

where $f_{k}^{i, j}$ are fitted parameters, $l$ is $1(0)$ when $j$ is even (odd), and $l_{i}$ is $i-8$ for the three bond lengths and $i-11$ for the three bond angles. For the angle $q_{14}=\beta_{1}, g_{j}^{i}(\tau)$ is given by:

$$
g_{j}^{i}(\tau)=\sum_{k=1}^{m} f_{k}^{i, j} \sin (2 k+l) \tau,
$$

where $f_{k}^{i, j}$ are fitted parameters and $l$ is $-1(0)$ when $j$ is even (odd). At last, for the angle $q_{15}=\beta_{2}, g_{j}^{i}(\tau)$ is given by:

$$
g_{j}^{i}(\tau)=\sum_{k=0}^{m} f_{k}^{i, j} \cos (2 k+l) \tau
$$


where $f_{k}^{i, j}$ are fitted parameters and $l$ is $1(0)$ when $j$ is even (odd).

\section{Appendix B: Exact bending-torsion-rotation Hamiltonian}

When using a reference configuration characterized by rigid linear NCO group and $C_{3 v}$ methyl group, the following expressions can be obtained for the nonvanishing inverse inertia tensor components:

$$
\begin{aligned}
\mu_{t t} & =4\left(c-p_{0} t\right)\left(1-t^{2}\right) / D_{t}, \\
\mu_{y y} & =\left(c+p_{0} t\right) / D_{t}, \\
\mu_{t y} & =2 d \sqrt{1-t^{2}} / D_{t}, \\
\mu_{\tau \tau} & =\left[\left(c^{2}-d^{2}-p_{0}^{2}-2 I_{z}^{2} d\right)\left(1-t^{2}\right) / I_{z}^{2}\right. \\
& \left.+2 c\left(1+t^{2}\right)-4 t p_{0}\right] / D_{\tau}, \\
\mu_{x \tau} & =\sqrt{2(1+t)}\left[d-c+p_{0}-t\left(d+c-p_{0}\right)\right] / D_{\tau}, \\
\mu_{z \tau} & =\sqrt{2(1-t)}\left[d-c-p_{0}+t\left(d+c+p_{0}\right)\right] / D_{\tau}, \\
\mu_{x x} & =2(1+t)\left(c-p_{0}\right) / D_{\tau}, \\
\mu_{x z} & =-2 \sqrt{1-t^{2}} d / D_{\tau}, \\
\mu_{z z} & =2(1-t)\left(c+p_{0}\right) / D_{\tau},
\end{aligned}
$$

where:

$$
\begin{aligned}
D_{t}= & c^{2}-d^{2}-p_{0}^{2} t^{2}, \\
D_{\tau}= & \left(c^{2}-d^{2}-p_{0}^{2}\right)\left(1-t^{2}\right), \\
c= & I^{1}+I_{x}^{2}+\left[\left(m_{1}+m_{2}\right)\left(m_{3}+m_{4}+3 m_{\mathrm{H}}\right) r_{1}^{2}\right. \\
& \left.+\left(m_{1}+m_{2}+m_{3}\right)\left(m_{4}+3 m_{\mathrm{H}}\right) r_{2}^{2}\right] / M, \\
d= & I^{1}-I_{x}^{2}+\left[\left(m_{1}+m_{2}\right)\left(m_{3}+m_{4}+3 m_{\mathrm{H}}\right) r_{1}^{2}\right. \\
& \left.-\left(m_{1}+m_{2}+m_{3}\right)\left(m_{4}+3 m_{\mathrm{H}}\right) r_{2}^{2}\right] / M, \\
p_{0}= & 2\left(m_{1}+m_{2}\right)\left(m_{4}+3 m_{\mathrm{H}}\right) r_{1} r_{2} / M, \\
r_{1}= & {\left[m_{1} r_{a}+m_{2} r\left(\mathrm{C}_{\alpha} \mathrm{N}\right)\right] /\left(m_{1}+m_{2}\right), } \\
r_{2}= & {\left[m_{4} r(\mathrm{CN})+3 m_{\mathrm{H}} r_{d}\right] /\left(m_{4}+3 m_{\mathrm{H}}\right), } \\
r_{a}= & r(\mathrm{CO})+r\left(\mathrm{C}_{\alpha} \mathrm{N}\right), \\
r_{d}= & r(\mathrm{CN})+r(\mathrm{CH}) \cos \beta, \\
I^{1}= & m_{1} m_{2} /\left(m_{1}+m_{2}\right) r(\mathrm{CO})^{2}, \\
I_{x}^{2}= & 3 m_{\mathrm{H}} r(\mathrm{CH})^{2}\left[\frac{1}{2} \sin ^{2} \beta+m_{4} /\left(m_{4}+3 m_{\mathrm{H}}\right) \cos ^{2} \beta\right], \\
I_{z}^{2}= & 3 m_{\mathrm{H}} r(\mathrm{CH})^{2} \sin ^{2} \beta,
\end{aligned}
$$

with $\beta=\angle \mathrm{NC}_{\alpha} \mathrm{H}$ and $m_{1}, m_{2}, m_{3}$, and $m_{4}$ being shorthand notations for $m_{\mathrm{O}}, m_{\mathrm{C}}, m_{\mathrm{N}}$, and $m_{\mathrm{C}_{\alpha}}$, respectively. Equations (B1) and (B2) lead to the following values for $A$ and $B$ in Eqs. (9):

$$
\begin{aligned}
& A=\frac{-d}{c^{2}-d^{2}-p_{0}^{2}}, \\
& B=\frac{2\left(c+p_{0}\right)}{c^{2}-d^{2}-p_{0}^{2}} .
\end{aligned}
$$

[1] W. Worthy, Chem. \& Engineering News Archive 63, 27 (1985).

[2] J. A. Duckett, A. G. Robiette, and I. M. Mills, J. Mol. Spectrosc. 62, 34 (1976).

[3] S. Cradock, J. Mol. Spectrosc. 92, 170 (1982).
[4] A. Wierzbicki, J. Koput, and M. Krȩglewski, J. Mol. Spectrosc. 99, 102 (1983)

[5] M. Krȩglewski and P. Jensen, J. Mol. Spectrosc. 103, 312 (1984).

[6] J. Koput, J. Mol. Spectrosc. 104, 12 (1984). 
[7] M. Krȩglewski, J. Mol. Spectrosc. 105, 8 (1984).

[8] J. Koput, J. Mol. Spectrosc. 106, 12 (1984).

[9] J. Koput, J. Mol. Spectrosc. 115, 131 (1986).

[10] J. Koput, J. Mol. Spectrosc. 118, 448 (1986).

[11] J. Koput, J. Mol. Spectrosc. 127, 51 (1988).

[12] R. G. Lett and W. H. Flygare, J. Chem. Phys. 47, 4730 (1967).

[13] R. F. Curl, V. M. Rao, K. V. L. N. Sastry, and J. A. Hodgeson, J. Chem. Phys. 39, 3335 (1963).

[14] J. Cernicharo, Z. Kisiel, B. Tercero, L. Kolesniková, I. R. Medvedev, A. López, S. Fortman, M. Winnewisser, F. C. de Lucia, J. L. Alonso, and J.-C. Guillemin, A\&A 587, L4 (2016).

[15] D. T. Halfen, V. V. Ilyushin, and L. M. Ziurys, Astrophys. J. 812, L5 (2015).

[16] F. Goesmann, H. Rosenbauer, J. H. Bredehöft, M. Cabane, P. Ehrenfreund, T. Gautier, C. Giri, H. Krüger, L. Le Roy, A. J. MacDermott, S. McKenna-Lawlor, U. J. Meierhenrich, G. M. M. Caro, F. Raulin, R. Roll, A. Steele, H. Steininger, R. Sternberg, C. Szopa, W. Thiemann, and S. Ulamec, Science 349, 497 (2015).

[17] K. Altwegg, H. Balsiger, J. J. Berthelier, A. Bieler, U. Calmonte, S. Fuselier, F. Goesmann, S. Gasc, T. I. Gombosi, L. Le Roy, J. de Keyser, A. Morse, M. Rubin, M. Schuhmann, M. G. G. T. Taylor, C.-Y. Tzou, and I. Wright, MNRAS 469, S130 (2017).

[18] R. P. Hirschmann, R. N. Kniseley, and V. A. Fassel, Spectrochim. Acta. 21, 2125 (1965).

[19] J. F. Sullivan, H. L. Heusel, W. M. Zunic, J. R. Durig, and S. Cradock, Spectrochim. Acta. A: Molecular Spectroscopy 50, 435 (1994).

[20] I. Tokue, A. Hiraya, and K. Shobatake, Chem. Phys. 117, 315 (1987).

[21] S. Cradock, E. A. V. Ebsworth, and J. D. Murdoch, J. Chem. Soc. Faraday Trans. 2 68, 86 (1972).

[22] T. Pasinszki, T. Veszprèmi, M. Fehèr, B. Kovac̀, L. Klasinc, and S. P. McGlynn, Int. J. Quantum Chem. 44(S26), 443 (1992).

[23] D. Lauvergnat, A. Nauts, Y. Justum, and X. Chapuisat, J. Chem. Phys. 114, 6592 (2001).

[24] D. Lauvergnat and A. Nauts, Chem. Phys. 305, 105 (2004).

[25] J. H. D. Eland, Phil. Trans. Roy. Soc. Lond. A 268, 87 (1970).

[26] D.-H. Han, P. G. Pearson, and T. A. Baillie, J. Lab. Comp. Radiopharm. 27, 1371 (1989).
[27] B. Maté, G. Molpeceres, V. Timón, I. Tanarro, R. Escribano, J. C. Guillemin, J. Cernicharo, and V. J. Herrero, MNRAS 470, 4222 (2017).

[28] X. Tang, G. A. Garcia, J.-F. Gil, and L. Nahon, Rev. Sci. Instrum. 86, 123108 (2015).

[29] L. Nahon, N. de Oliveira, G. A. Garcia, J.-F. Gil, B. Pilette, O. Marcouillé, B. Lagarde, and F. Polack, J. Synchrotron Radiat. 19, 508 (2012).

[30] B. Mercier, M. Compin, C. Prevost, G. Bellec, R. Thissen, O. Dutuit, and L. Nahon, J. Vac. Sci. \& Technol. A 18, 2533 (2000).

[31] G. A. Garcia, B. K. de Miranda, M. Tia, S. Daly, and L. Nahon, Rev. Sci. Instrum. 84, 053112 (2013).

[32] J. C. Poully, J. P. Schermann, N. Nieuwjaer, F. Lecomte, G. Grgoire, C. Desfranois, G. A. Garcia, L. Nahon, D. Nandi, L. Poisson, and M. Hochlaf, Phys. Chem. Chem. Phys. 12, 3566 (2010).

[33] F. Merkt, A. Osterwalder, R. Seiler, R. Signorell, H. Palm, H. Schmutz, and R. Gunzinger, J. Phys. B 31, 1705 (1998).

[34] E. B. Wilson, J. C. Decius, and P. C. Cross, Molecular vibrations (McGraw-Hill Book Company, Inc., 1955).

[35] A. Kapur, P. G. Mezey, and R. Steer, Chem. Phys. Lett. 78, 81 (1981).

[36] H.-J. Werner, P. J. Knowles, G. Knizia, F. R. Manby, and M. Schütz, WIREs Comput. Molec. Sci. 2, 242 (2012).

[37] L. H. Coudert, J. Mol. Spectrosc. 154, 427 (1992).

[38] L. H. Coudert, J. Mol. Spectrosc. 165, 406 (1994).

[39] L. H. Coudert, J. Mol. Spectrosc. 181, 246 (1997).

[40] R. Lanquetin, L. H. Coudert, and C. Camy-Peyret, J. Mol. Spectrosc. 195, 54 (1999).

[41] L. H. Coudert, Mol. Phys. 96, 941 (1999).

[42] L. H. Coudert, J. Mol. Spectrosc. 330, 112 (2016).

[43] J. T. Hougen, P. R. Bunker, and J. W. C. Johns, J. Mol. Spectrosc. 34, 136 (1970).

[44] M. A. Mekhtiev, P. D. Godfrey, and V. Szalay, J. Mol. Spectrosc. 180, 42 (1996).

[45] L. H. Coudert, B. Gans, G. A. Garcia, and J.-C. Loison, J. Chem. Phys. 148, 054302 (2018).

[46] P. T. Ruhoff and M. A. Ratner, Int. J. Quant. Chem. 77, 383 (2000).

[47] F. Duschinsky, Acta Physicochim. URSS 7, 551 (1937).

[48] A. Capobianco, R. Borrelli, C. Noce, and A. Peluso, Theor. Chem. Acc. 131, 1181 (2012).

[49] T. Baer, Annu. Rev. Phys. Chem. 40, 637 (1989).

[50] R. Signorell and F. Merkt, Faraday Discuss. 115, 205 (2000). 